\title{
The Taitao ophiolite-granite complex, Chile: Emplacement of ridge-trench intersection oceanic lithosphere on land and the origin of calc-alkaline I-type granites
}

\author{
${ }^{1}$ Research Institute for Humanity and Nature, Motoyama 457-4, Kamigamo, Kita-Ku, Kyoto 603-8047, Japan \\ ${ }^{2}$ University of Tsukuba, Ten-nodai 1-1-1, Tsukuba 305-8572, Japan. Corresponding author E-mail: amma.ryo.ge@u.tsukuba.ac.jp \\ ${ }^{3}$ Earthquake Research Institute, University of Tokyo, Yayoi 1-1-1, Bunkyo-Ku, Tokyo 113-0032, Japan \\ * Present address: Medical Device Supply Chain Asia Pacific, Johnson \& Johnson K.K. Medical Company, 3-5-2 Nishikanda, \\ Chiyoda-ku, Tokyo 101-0065, Japan
}

DOI: 10.18814/epiiugs/2015/v38i4/82424

The late Miocene - early Pliocene Taitao ophiolite is exposed $\sim 30 \mathrm{~km}$ southeast of the Chile triple junction, where a spreading center of the Chile ridge system is subducting underneath the South America plate. This unique tectonic setting provides an excellent opportunity to study the emplacement mechanism of a ridge-trench intersection ophiolite and the complex magmatic interactions between the subducting ridge, overlying crust and sediments, and the mantle wedge. As a result of these interactions, several granitic plutons were formed contemporaneously with emplacement of the ophiolite. We review previous studies of the Taitao ophiolite and use new geochemical data to discuss the mechanism that formed juvenile magma of calc-alkaline I-type granites during ridge subduction. Our model implies that the magmas of the Taitao granites formed due to partial melting of hot oceanic crust adjacent to the subducting mid-oceanic ridge that has been contami-nated by deep crustal material and/or metasomatized sub-arc mantle. The partial melting took place under garnet-free amphibolite conditions. The juvenile magmas then incorporated different amounts of subducted sediments and/or continental material to form the I-type granites with various compositions. Subduction of fracture zones played an important role in these processes.

\section{Introduction}

Ophiolites are regarded as pieces of ancient oceanic lithosphere emplaced onto continental crust (Anonymous, 1972). Once it was presumed that this allochthonous material invariably formed at a midocean spreading ridge. Today, most ophiolites are thought to form in supra-subduction zone (SSZ) settings in an island-arc or back-arc spreading environment (Miyashiro, 1973; Pearce and Robinson, 2010; Dilek and Furnes, 2011, 2014). However, even for extensively exposed and intensively studied examples like the Semail ophiolite, Oman, disagreement persists between ridge-origin scholars (Boudier et al., 1996; MacLeod and Yaouancq, 2000; Miyashita et al., 2003; Adachi and Miyashita, 2003; Boudier and Nicolas, 2011) and SSZ-origin scholars (Dilek and Flower, 2003; Dilek and Furnes, 2009; Dilek and Thy, 2009; Pearce and Robinson, 2010). One confounding factor is that all ophiolites must have experienced modification in SSZ environments just before their emplacement that may have obscured or obliterated primary features formed during their seafloor spreading origin (Tsuchiya et al., 2013; Kanke and Takazawa, 2014; Yoshikawa et al., 2015). Another factor may be that tectonic settings at the times of ophiolite formation and emplacement are ambiguous (Dilek and Robinson, 2003; Warren et al., 2005).

The Taitao ophiolite, southern Chile (Fig. 1), is one of the few ophiolites for which the tectonic setting is clearly understood, because of its young age (<6 Ma: Anma et al., 2006) and the nearby presence of a subducting spreading-ridge segment of the Chile ridge system (Cande et al., 1982, 1987; Cande and Leslie, 1986; Behrmann et al., 1994). The plate configuration and plate kinematics before and after the oceanic lithosphere formed and was emplaced as an ophiolite can be well reconstructed from various data.

The thermal history of an ophiolite can be prolonged, especially one that formed at a spreading ridge far from land in a large ocean, migrated toward a continental margin and emplaced in a SSZ environment. However, in the case of the Semail ophiolite, its generation and emplacement took place in a short period: trondhjemite in the Semail ophiolite yielded zircon U-Pb ages of $95.3 \mathrm{Ma}$, whereas amphibolite in the metamorphic sole yielded zircon U-Pb ages of $244.5 \mathrm{Ma}$ (Warren et al., 2005). Tsuchiya et al. (2013) 
also reported similar ages $(\sim 100 \mathrm{Ma})$ in both the quartz diorite that formed at the ridge axis and the late-stage tonalite that represents arc-like magmatism. However, progressive metamorphism continued after emplacement as obduction and subduction proceeded, and the peak metamorphism that resulted in eclogite formation took place at $\sim 79 \mathrm{Ma}$ (Warren et al., 2005). Exhumation of blueschist in the southern part of the Semail ophiolite resulted in cooling of these rocks to the closing temperature of the zircon fission-track system $\left(\sim 250^{\circ} \mathrm{C}\right)$ at $266-70 \mathrm{Ma}$ (Yamato et al., 2007). Jacobs et al. (2015) reported apatite fission-track and zircon and apatite (U-Th)/He ages of igneous rocks and the metamorphic sole from the section of the Semail ophiolite exposed in the UAE. They showed that the plutonic rocks were cooled to the closing temperatures of two of these three systems (zircon (U-Th)/He and apatite fission track) within 10 to $30 \mathrm{~m}$ (84.564.7 Ma and 87-62 Ma, respectively) of crust formation, whereas exhumation of metamorphic rocks took a longer period of time (until 63-31 Ma according to apatite fission-track ages). Both the plutonic and metamorphic rocks were cooled to the closing temperature of the apatite (U-Th)/He system in Miocene time (Jacobs et al., 2015). Thus, during its prolonged thermal history, various modification processes could have affected the Semail ophiolite. This history can be compared with that, which is much more precisely determined for the Taitao ophiolite. It took only $1 \mathrm{my}$ from the formation of the oceanic lithosphere through its emplacement and exhumation, as detailed in this paper (Fig. 1d).

The formation, emplacement and exhumation processes of ophiolites generate a wide range of geochemical variations (Flower and Dilek, 2003; Balestro et al., 2015; Fareeduddin and Dilek, 2015, Saccabu et al., 2015). In the case of the Semail ophiolite, the plutonic rocks range in chemical composition from $\sim 48 \mathrm{wt} . \% \mathrm{SiO}_{2}$ in oceanic massive gabbro to $\sim 75 \mathrm{wt} . \% \mathrm{SiO}_{2}$ in late-stage tonalite (Tsuchiya et al., 2013). The chemical variation of the Taitao ophiolite-granite complex is similar, ranging from 48 to $74 \mathrm{wt} . \% \mathrm{SiO}_{2}$ for the plutonic rocks. However, in the Semail ophiolite, the late-stage felsic plutonic rocks are minor components. In contrast, in the Taitao ophiolite these rocks occupy as much as $10 \%$ of the exposure (Fig. 1c).

The advantage of studying the Taitao ophiolite and its related igneous rocks is that this area has a complete record of the magmatic history and the full range of chemical variation seen in ophiolites, with better tectonic and geochronological constraints than any other example in the world. Evidence of every process, from crustal formation to ophiolite emplacement, that took place over the span of 1 my was "frozen" into the geological record of this area. The Taitao ophiolite provides an excellent opportunity to study the emplacement mechanism of a ridge-trench intersection ophiolite, with its complex magmatic interactions between the subducting ridge, the overlying crust and sediments, and the mantle. Among these interactions, the mechanism that created voluminous intermediate to felsic magma in this area is of fundamental importance for understanding the processes that create continental crust (Kon et al., 2013). In this study, we used chemical compositions and the $\mathrm{Sr}-\mathrm{Nd}$ isotope system to ascertain the magmatic interactions during the formation and emplacement of the Taitao ophiolite.

\section{Tectonic setting of the Taitao ophiolite and related rocks}

The Taitao ophiolite is $\sim 30 \mathrm{~km}$ southeast of the Chile triple junction, where a segment of the spreading Chile ridge system is subducting underneath the South America plate off the Taitao Peninsula, the westernmost promontory of the Chilean coast. The Chile ridge system, which separates the Nazca plate to the north and the Antarctica plate to the south, has a central axis trending northnorthwest that consists of several segments separated by transform fracture zones trending east-northeast (Fig. 1a). The convergent rates of the Nazca and Antarctica plates with respect to the South America plate are $9 \mathrm{~cm} / \mathrm{y}$ and $2 \mathrm{~cm} / \mathrm{y}$, respectively (Herron et al., 1981; Cande et al. 1982; Tebbens and Cande, 1997; Tebbens et al., 1997). The current plate configuration and plate kinematic data (Cande and Leslie 1986; Breitspecher and Thorkelson, 2009) allow us to deduce that short segments of this spreading center $(<100 \mathrm{~km} \mathrm{long})$ began subducting off the Taitao Peninsula at $\sim 6 \mathrm{Ma}$ and $\sim 3 \mathrm{Ma}$. The formation and emplacement of the Taitao ophiolite are related to the first of these subduction events (Anma et al., 2006).

The study area, in the westernmost part of the Taitao Peninsula, exposes four major rock suites (Fig. 1b): the Chonos metamorphic complex, the Chile Margin Unit (CMU), the Taitao ophiolite, and the Taitao granites (Forsythe et al., 1986; Nelson et al., 1993; Bourgois et al., 1992; 1993; Guivel et al., 1999). The Chonos metamorphic rocks, which mostly originated from siliciclastic and volcaniclastic protoliths, form the basement of the study area. Thomson and Herve (2002) determined Late Permian to Jurassic (258 to $207 \mathrm{Ma}$ ) fissiontrack and $\mathrm{U}-\mathrm{Pb}$ ages from zircons in the basement rocks in the forearc region.

\section{Chile Margin Unit}

The CMU is a mixture of lava, pyroclastic rocks and semiconsolidated sediments (Fig. 1b, c). It was first defined by Bourgois et al. (1993) to distinguish it from the Main Volcanic Unit (see next section). The CMU directly overlies the pre-Jurassic basement, is of lower metamorphic grade, and appears to have deposited in the forearc area of the Chilean margin. Whole-rock K-Ar ages for the CMU range from 4.4 to $2.5 \mathrm{Ma}$ (Mpodozis et al., 1985). Volcanic rocks of the CMU have rather enriched compositions comparing with normal midocean ridge basalt (N-MORB) (Lagabrielle et al., 1994; Le Moigne et al., 1996; Guivel et al., 1999).

Anma and Orihashi (2013) dredged pyroclastic rocks resembling those of the CMU from the South Taitao Ridge, offshore from the Taitao ophiolite (Fig. 1b), and argued that the magmatic activity of the CMU started there at 5.2 Ma, migrated eastward at a rate of 2 to $5 \mathrm{~cm} / \mathrm{y}$, forming the CMU (Fig. 1c, d), and continued to the Pan de Azucar region (PA in Fig. 1b) where volcanic activity took place at 4.4 Ma based on zircon U-Pb data. All of these rocks contained inherited zircons that originated from subducted sediments. Anma and Orihashi (2013) suggested that the magmatic activity of the CMU is related to the subduction of a fracture zone separating segments of the Chile ridge system.

\section{Taitao ophiolite}

The Taitao ophiolite exposes the complete sequence of a Penrosetype oceanic lithosphere (Anonymous, 1972) (Fig. 1c). The top part, in the north, is represented by pillow lava and sheet flows interbedded with clastic deposits, which we refer to as the Main Volcanic Unit (MVU) after Bourgois et al. (1993). To the south are sheeted dikes, gabbros and ultramafic rocks (Forsythe et al. 1986; Kaeding et al., 

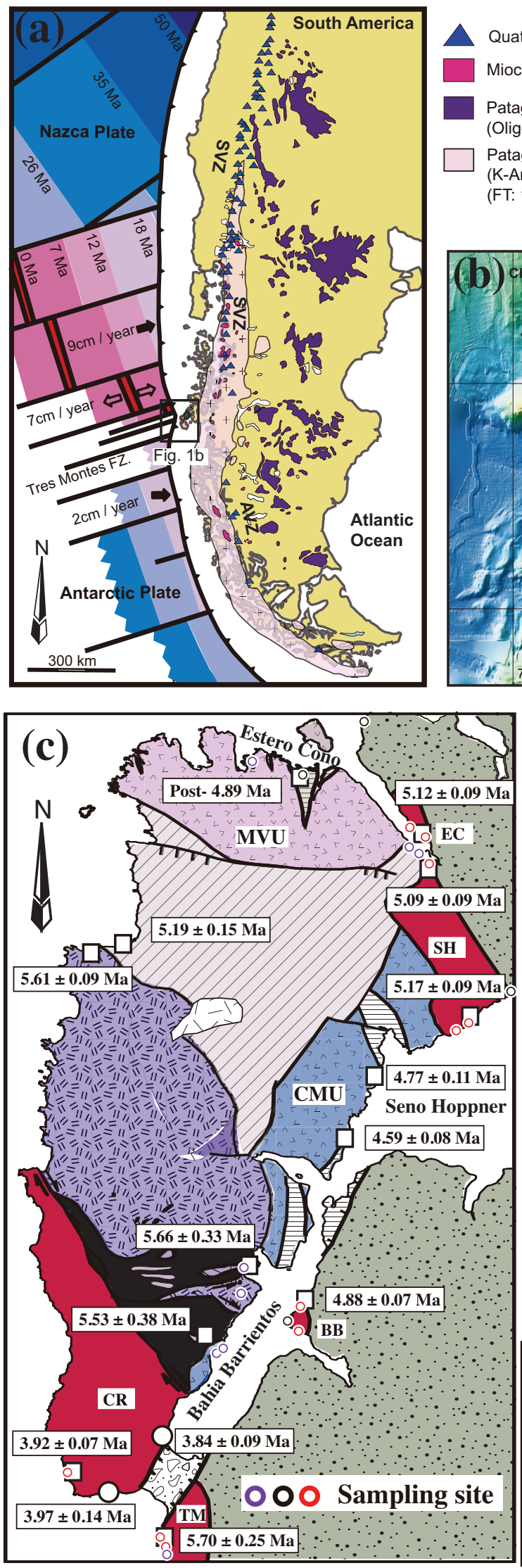

Quaternary volcanoes

Miocene granites

Patagonian volcanic field (Oligocene-Pleistocene)

Patagonian batholith (K-Ar: $155 \mathrm{Ma}-92 \mathrm{Ma})$ (FT: $127 \mathrm{Ma}-39 \mathrm{Ma}$ )
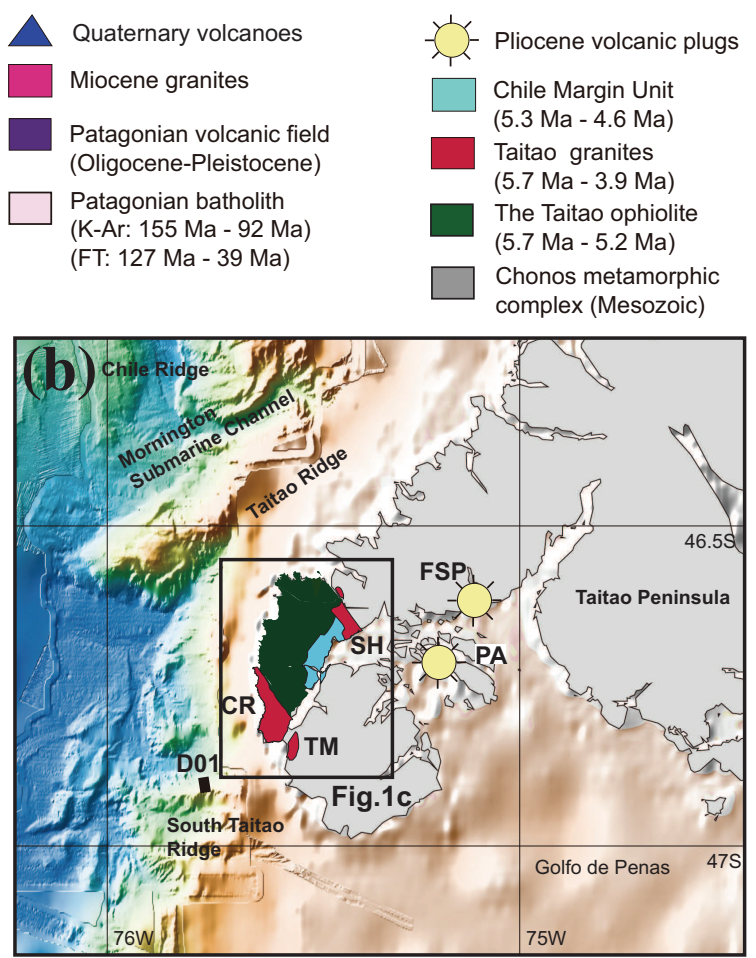

(d) Chile Margin Unit

\begin{tabular}{|c|c|}
\hline \multicolumn{2}{|c|}{ Taitao Ophiolite } \\
\hline TPB338 $\square$ & Detrital zircon from MVU \\
\hline TPG262 $\square$ & Sheeted Dike Complex \\
\hline $\begin{array}{l}\text { TPG107 } \\
\text { TPG244 }\end{array}$ & Gabbro \\
\hline TPB268 & \\
\hline
\end{tabular}

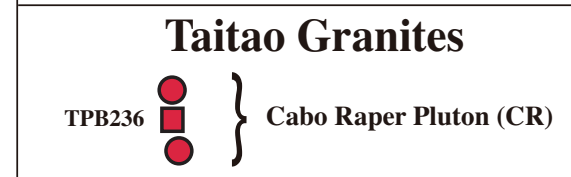

TPB246 $\square \quad$ Bahia Barrientos Pluton (BB)

TPD169 $\square \quad$ Estero Cono Pluton (EC)

$\left.\begin{array}{l}\text { TPD172 } \\ \text { TPD110 }\end{array}\right\}$ Seno Hoppner Pluton (SH)

TPA14 $\square$ Tres Montes Pluton (TM)

$$
\begin{array}{llllllll}
\hline 0 & 2 & 4 & 6 & 8 & 10 & 12 & 14 \\
\cline { 4 - 6 } & & & \text { Ages (Ma) } & \mathrm{O} & \text { Herve et al. (2003) }
\end{array}
$$

\begin{tabular}{|c|c|}
\hline Holocene & The Taitao Ophiolite \\
\hline Cor. Conglomerates & 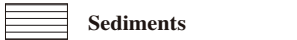 \\
\hline The Taitao Granites & Pillow lava/sheet flow \\
\hline Granitic rocks & a \\
\hline $\begin{array}{l}\text { Chile Margin Unit } \\
\text { Pillow lava/pyroclasts } \\
\text { interbeded with sediments }\end{array}$ & W/ Sheeted dike complex \\
\hline Basement (Pre-Jurassic) & Ultramafic racks \\
\hline$\because \quad$ Meta-sedimentary rocks & Ultramafic rocks \\
\hline
\end{tabular}

Figure 1. (a) Tectonics of the Chile Ridge subduction zone. (b) Distribution of the Taitao ophiolite and related igneous rocks together with detailed bathymetry. (c) Bedrock geology of the study area, modified from Anma et al. (2006), showing distribution of sampling sites for this study. MUV: Main Volcanic Unit, CMU: Chile Margin Unit, EC: Estero Cono Pluton, SH: Seno Hoppner pluton, BB: Bahia Barrientos intrusion, CR: Cabo Raper pluton, TM: Tres Montes pluton. (d) U-Pb age distribution of samples in this study. 
1990; Nelson et al., 1993; Bourgois et al., 1993). The ophiolite shows evidence of hydrothermal alteration that is typical of ocean-floor metamorphism (Shibuya et al., 2007). The ultramafic rocks provide evidence of at least two stages of melting, one that took place at $\sim 1.6 \mathrm{Ga}$ and the other of recent age related to magmatism in the Chile ridge (Schulte et al., 2009). The gabbros have compositions similar to N-MORB, whereas the pillow basalts of the MVU have enriched compositions (Lagabrielle et al., 1994; Le Moigne et al., 1996; Guivel et al., 1999) and isotopic compositions (Kaeding et al., 1990) that suggest crustal contamination. Because of this compositional difference, the plutonic section has been referred to separately as the Bahia Barrientos ophiolite (Lagabrielle et al., 1994; Le Moigne et al., 1996; Guivel et al., 1999). However, Schulte et al. (2009) reported that the ultramafic rocks and gabbros of the plutonic section also have a range of chemical and isotopic compositions that could be explained in terms of seawater contamination (for $\mathrm{Sr}$ isotopic ratios) and incorporation of sediment and basement rocks into the melt source of the Taitao ophiolite. Anma et al. (2006) explained the enriched composition of the MVU in terms of alteration along fracture zones or sub-segment boundaries, as reported previously from the Chile ridge (Klein and Karsten, 1995; Karsten et al., 1996; Sherman et al., 1997; Strum et al., 1999, 2000), and considered the MVU to be a member of the Taitao ophiolite.

Previously reported $\mathrm{U}-\mathrm{Pb}$ ages of the gabbros and sheeted dikes are $~ 5.6 \mathrm{Ma}$ and $~ 5.2 \mathrm{Ma}$, respectively (Fig. 1c, d; Anma et al., 2006). The sheeted dikes were thermally metamorphosed by the intrusion of the 5.1 Ma Seno Hoppner pluton (SH in Fig. 1c) (Shibuya et al., 2007). Thus, the center of magmatic activities that formed the ophiolite migrated from south to north as a segment of the Chile ridge subducted obliquely against the Chilean continental margin (Anma et al., 2006, 2009). Anma et al. (2009) reported U-Pb ages of zircons in clastic sediments in the MVU, of which 19 grains out of 24 yielded ages younger than $5.6 \mathrm{Ma}$, and concluded that their deposition postdated $\sim 4.9 \mathrm{Ma}$. Veloso et al. (2007) argued that these clastic materials were derived from the south-southeast based on sedimentary structures and anisotropy of magnetic susceptibility fabrics. Thus, the post-5.6 Ma clastic zircons must have been derived from the plutonic section of the Taitao ophiolite and the Taitao granites, and by the time of deposition, most of these plutonic rocks must have been exposed subaerially (Anma et al., 2009). Zircon fission-track ages from gabbros of the ophiolite that coincided with the U-Pb ages also attest to rapid exhumation and cooling of the plutonic section (Anma et al., 2006).

The gabbros continued to fold even below their Curie temperature, as evidenced by macroscopic folding and Z-shaped demagnetization paths in the layered gabbros (Anma et al., 2005; Veloso et al., 2005), whereas the sheeted dike complex and the MVU rotated as rigid blocks as deformation proceeded (Veloso et al., 2005, 2009). It was the eastern part of the subducted segment of the Chile ridge that was emplaced as the plutonic section of the Taitao ophiolite (Veloso et al., 2005; Anma et al., 2005, 2006). As the spreading center subducted obliquely, the center of magmatic activities migrated northward. During the time the midsection of the MVU was deposited, the plutonic section must have been subaerially exposed, supplying sediment to the subducting central valley (Anma et al., 2006, 2009).

\section{Taitao granites}

The term "Taitao granites" collectively denotes the felsic plutonic rocks distributed around the Taitao ophiolite. These four plutons and a small body of granitic rocks exposed along the Bahia Barrientos (Fig. 1c) have zircon U-Pb ages that range from 5.7 Ma to $3.9 \mathrm{Ma}$ (Fig. 1d) and compositions that range from trondhjemitic to granitic and tonalitic to granodioritic (Fig. 2b; Anma et al., 2009). The plutons are the Tres Montes pluton (TM in Fig, 1c; $5.7 \mathrm{Ma}$ ) and Cabo Raper pluton (CR; 3.9 Ma) to the south of the Taitao ophiolite and the Seno Hoppner (SH) and Estero Cono (EC) plutons (5.2$5.1 \mathrm{Ma}$ ) to the northeast (Fig. 1). The Bahia Barrientos granite (BB in Fig. 1c; $4.9 \mathrm{Ma}$ ), intruded along the edge of the CMU, is thought to be related to CMU magmatism on the basis of its age (Anma and Orihashi, 2013).

The origin of the Taitao granites is controversial. Kaeding et al. (1990) were the first to recognize that the Cabo Raper pluton has a tonalitic to granodioritic composition with calc-alkaline I-type affinity, whereas the Seno Hoppner pluton has characteristics of S-type affinity. In contrast, Bourgois et al. (1996) suggested that the Cabo Raper pluton is a heavy rare earth element (HREE)-depleted trondhjemitetonalite-granodiorite (TTG) that formed by slab melting under conditions of the amphibolite-eclogite transition. Guivel et al. (1999) reported that the Seno Hoppner pluton differs from the Cabo Raper and Seno Hoppner plutons in having a trondhjemite-granite composition and characteristics, typical of the calc-alkaline series. They also suggested that the Cabo Raper and Tres Montes plutons formed by slab melting under amphibolite-eclogite transition conditions.

Kon et al. (2013) recognized hornblende-bearing and hornblendefree members in the Seno Hoppner and Cabo Raper plutons. The hornblende-free members have trondhjemitic to granitic compositions whereas the hornblende-bearing members plot in the tonalitic to granodioritic compositional field along with the hornblende-bearing Cabo Raper pluton. The hornblende-free members have a pronounced negative Eu anomaly whereas the hornblende-bearing members have none or a small Eu anomaly. On the basis of trace-element compositions and tectonic constraints, they suggested that these granites were generated by partial melting of subducted oceanic crust with garnet-free-amphibolite conditions at depths shallower than $30 \mathrm{~km}$.

The Tres Montes granodiorite includes numerous inherited zircons (7 of 15) with an age distribution similar to those from the CMU, whereas inherited zircons are rare in the Seno Hoppner and Cabo Raper granites (Herve et al., 2003; Anma et al., 2009). Thus, it has been proposed that the Tres Montes pluton originated with a strong influence from subducted sediments, whereas the Seno Hoppner and Cabo Raper plutons formed from juvenile granitic magmas (Anma $e t$ al., 2009; Kon et al., 2013). Kaeding et al. (1990) argued from Sr$\mathrm{Nd}$ isotopic compositions that the Seno Hoppner and Cabo Raper granites formed by assimilation of basaltic magmas with 5-15\% forearc sediments or metamorphic basement rocks.

\section{Scope and analytical methods}

To investigate the complicated magmatic processes resulting from the interaction between the spreading ridge and the subduction zone, and to test previous hypotheses, we conducted $\mathrm{Sr}-\mathrm{Nd}$ isotopic analyses of this ridge-trench intersection ophiolite (Wakabayashi and Dilek, 2003; Dilek and Furnes, 2011, 2014) and its related rocks. The analyzed samples are described in Table 1 and their locations shown by circles in Figure 1c. Apart from 5 ophiolite and 10 granite samples, we measured two mafic volcanic rocks from the CMU and two mafic 
Table 1. Geological unit and rock description of this study

\begin{tabular}{|c|c|c|c|c|c|}
\hline \multicolumn{2}{|r|}{ Geological unit } & Unit description & Sample ID & Age $(\mathrm{Ma})^{*}$ & Rock description \\
\hline \multirow{2}{*}{\multicolumn{2}{|c|}{$\begin{array}{l}\text { Mafic dikes in granite } \\
\text { plutons }\end{array}$}} & Estero Cono (EC) dike & TPD185 & $<5.2$ & basaltic dike intruded into the Seno Hoppner pluton \\
\hline & & Tres Montes (TM) dike & TPA009 & $<5.7$ & syn-plutonic basaltic dike intruded into the Tres Montes pluton \\
\hline \multirow{2}{*}{\multicolumn{2}{|c|}{$\begin{array}{l}\text { Chile Margin Unit } \\
\text { (CMU) }\end{array}$}} & \multirow{2}{*}{ Lavas and clastic rocks } & TPB174 & \multirow{2}{*}{$4.8 \sim 4.6$} & sheet flow: doleritic basalt \\
\hline & & & TPB175 & & glass-rich hyaloclastite \\
\hline \multirow{5}{*}{ 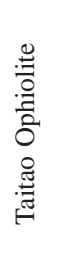 } & \multirow{2}{*}{$\begin{array}{l}\text { Main Volcanic } \\
\text { Unit (MVU) }\end{array}$} & \multirow{2}{*}{ Lavas and clastic rocks } & TPB345 & \multirow{2}{*}{$<5.2$} & basaltic pillow lava \\
\hline & & & ТPB347 & & basaltic massive sheet flow \\
\hline & $\begin{array}{l}\text { Estero Cono dike } \\
\text { complex }\end{array}$ & Sheeted dike complex & TPD208 & $<5.2$ & $\begin{array}{l}\text { dolerite to basalt, metamorphosed by the } \\
\text { intrusion of the Seno Hoppner pluton }\end{array}$ \\
\hline & \multirow{2}{*}{$\begin{array}{l}\text { Plutonic } \\
\text { complex }\end{array}$} & \multirow{2}{*}{$\begin{array}{l}\text { Gabbros and associated } \\
\text { doleritic dikes }\end{array}$} & ТРВ020 & \multirow[t]{2}{*}{5.6} & medium-grained gabbro (massive) \\
\hline & & & ТРВ024 & & fine-grained gabbro (mesocumulate) \\
\hline \multirow{10}{*}{ 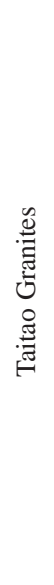 } & \multirow{2}{*}{$\begin{array}{l}\text { Tres Montes } \\
\text { (TM) pluton }\end{array}$} & \multirow{2}{*}{$\begin{array}{l}\text { Tonalite-granodiorite } \\
\text { with syn-plutonic dikes }\end{array}$} & TPA007 & \multirow{2}{*}{5.7} & $\begin{array}{l}\text { hybrid rocks formed due to syn-plutonic mafic dike } \\
\text { intrusion }\end{array}$ \\
\hline & & & TPA014 & & $\begin{array}{l}\text { fine to medium-grained biotite hornblende tonalite and } \\
\text { granodiorite }\end{array}$ \\
\hline & \multirow{3}{*}{$\begin{array}{l}\text { Seno Hoppner } \\
\text { (SH) pluton }\end{array}$} & \multirow{3}{*}{$\begin{array}{l}\text { Leucocratic granite with } \\
\text { mafic dike intrusion }\end{array}$} & TPD110 & \multirow{3}{*}{$5.2 \sim 5.1$} & \multirow{3}{*}{$\begin{array}{l}\text { medium-grained hornblende biotite granite, thermal aureole } \\
\text { contact in the ophiolite, mafic dike in the northern part. }\end{array}$} \\
\hline & & & TPD115 & & \\
\hline & & & TPD172 & & \\
\hline & \multirow{2}{*}{$\begin{array}{l}\text { Estero Cono } \\
\text { (EC) pluton }\end{array}$} & \multirow{2}{*}{ Tonalite-granodiorite } & TPD168 & \multirow{2}{*}{5.1} & \multirow{2}{*}{ fine to medium-grained biotite (hornblende) granodiorite } \\
\hline & & & TPD169 & & \\
\hline & \multirow{2}{*}{$\begin{array}{l}\text { Bahia Barrientos } \\
\text { (BB) pluton }\end{array}$} & \multirow{2}{*}{ Tonalite-trondhjemite } & TPB243 & \multirow{2}{*}{4.9} & \multirow{2}{*}{ fine to medium-grained biotite tonalite and trondhjemite } \\
\hline & & & ТРВ246 & & \\
\hline & $\begin{array}{l}\text { Cabo Raper } \\
\text { (CR) pluton }\end{array}$ & Tonalite-granodiorite & ТРВ236 & 3.9 & $\begin{array}{l}\text { fine to medium-grained biotite hornblende granodiorite } \\
\text { to tonalite }\end{array}$ \\
\hline \multirow{5}{*}{$\frac{\tilde{\omega}}{\tilde{0}}$} & Sediments & trench-slope sediments & TPB338 & $<4.9$ & turbiditic sediment in the MVU \\
\hline & \multirow{4}{*}{ Basement } & meta-igneous rocks & TPD095 & Jurassic** & felsic igneous rock (ryolitic)? \\
\hline & & \multirow{3}{*}{ meta-sedimentary rocks } & ТРВ240 & \multirow{3}{*}{ Jurassic** } & sandstone/mudstone alternation \\
\hline & & & ТРВ335 & & consolidated silici-clastic sediments \\
\hline & & & TPD099 & & pelitic schist \\
\hline
\end{tabular}

* Age data from Anma et al. (2006, 2009) and Anma and Orihashi (2013), ** from Thomson et al. (2002)

dikes intruded into the granite plutons. One of the granite samples (TPA007) was a hybrid rock mixed with a syn-plutonic basaltic dike. We also measured samples of basement rocks and turbiditic sediments to estimate the respective influences of contamination from Jurassic wall rocks and sediments.

Major- and trace-element concentrations were measured using an X-ray fluorescence spectrometer and a laser ablation inductively coupled plasma mass spectrometer at the Earthquake Research Institute using conventional methods (Tani et al., 2002; Orihashi and Hirata, 2003) (Table 2). The analytical results in Table 2 also include data for some granites reported in Anma et al. (2009). For wholerock $\mathrm{Sr}$ and $\mathrm{Nd}$ isotopic analyses, approximately $100 \mathrm{mg}$ of powdered sample was completely dissolved in a mixture of $38 \% \mathrm{HF}(1.0 \mathrm{ml})$, $68 \% \mathrm{HNO}_{3}(0.7 \mathrm{ml})$, and $70 \% \mathrm{HClO}_{4}(0.3 \mathrm{ml})$ at $140^{\circ} \mathrm{C}$ for $24 \mathrm{~h}$ using a hot plate. After evaporation, the residues were dissolved in 2 $\mathrm{ml}$ of $6 \mathrm{M} \mathrm{HCl}$ and heated at $100^{\circ} \mathrm{C}$ for $8 \mathrm{~h}$. Sr and REEs were separated by conventional ion-exchange chromatography. Separation of Nd from REEs was facilitated using Ln Resin (Eichrom Tec. Inc).

The purified $\mathrm{Sr}$ was loaded with a Ta activator on preconditioned $\mathrm{W}$ filaments and was measured in single filament mode. Nd isotope ratios were measured in Ta-Re double filaments mode. $\mathrm{Sr}$ and $\mathrm{Nd}$ isotopic ratios were determined using a TRITON mass spectrometer
(Thermo Fisher Scientific Co.) at the Research Institute for Humanity and Nature. Analytical precisions of isotopic ratio measurements are given as \pm 2 ó standard errors. The measured ${ }^{87} \mathrm{Sr} /{ }^{86} \mathrm{Sr}$ and ${ }^{143} \mathrm{Nd} /{ }^{144} \mathrm{Nd}$ ratios were normalized to ${ }^{86} \mathrm{Sr} /{ }^{88} \mathrm{Sr}=0.1194$ and ${ }^{146} \mathrm{Nd} /{ }^{144} \mathrm{Nd}=0.7219$, respectively. The ${ }^{87} \mathrm{Sr} /{ }^{88} \mathrm{Sr}$ value of the NIST SRM 987 reference material throughout the analyses was $0.710264 \pm 0.000014(2 \sigma, n=$ 14). The ${ }^{143} \mathrm{Nd} /{ }^{144} \mathrm{Nd}$ ratio of the La Jolla reference material was $0.511863 \pm 0.000015(2 \sigma, n=14)$. All samples were recalculated with reference to the values of reference materials. The results are listed in Table 3. We calculated the initial value of $\mathrm{Sr}$ and $\mathrm{Nd}$ isotope ratios (designated by the suffix $i$ ) using zircon $\mathrm{U}-\mathrm{Pb}$ ages reported by Anma et al. (2006, 2009). The basement rock isotopic ratios were calculated assuming the age of incorporation to be $5.5 \mathrm{Ma}$.

\section{Analytical results}

The analytical results for major and trace elements are shown in Figs. 2 to 6, and results for isotopic compositions are shown in Figs. 7 and 8. Our analyses in general are in good agreement with previously reported results.

The mafic rocks had a basaltic to basaltic-andesitic composition (Fig. 2a) that could be divided into N-type to E-type (enriched) MORB 
Table 2. Major and trace element compositions for the Taitao Ohpiolite, the Taitao granites, sediments and basement rocks

\begin{tabular}{|c|c|c|c|c|c|c|c|c|c|c|c|c|c|c|c|c|c|c|c|c|c|c|c|c|}
\hline \multirow{4}{*}{$\begin{array}{l}\text { Locality } \\
\text { Sample } \\
\text { No. }\end{array}$} & \multicolumn{5}{|c|}{ Taitao Ophiolite } & \multirow{2}{*}{\multicolumn{2}{|c|}{$\mathrm{CMU}$}} & \multirow{2}{*}{\multicolumn{2}{|c|}{$\begin{array}{c}\text { mafic dikes } \\
\text { in granites }\end{array}$}} & \multicolumn{10}{|c|}{ Taitao Granites } & \multirow{3}{*}{$\begin{array}{c}\text { Sediment } \\
\text { TPB }\end{array}$} & \multicolumn{4}{|c|}{ Basement } \\
\hline & \multicolumn{2}{|c|}{ MVU } & \multirow{2}{*}{$\begin{array}{r}\text { EC } \\
\text { TPD }\end{array}$} & \multicolumn{2}{|c|}{ gabbro } & & & & & & TM & & SH & & & $\mathrm{EC}$ & & B & CR & & & & & \\
\hline & ТPB & ТРВ & & TPB & TPB & \multirow{2}{*}{$\begin{array}{r}\text { TPB } \\
174\end{array}$} & \multirow{2}{*}{$\begin{array}{l}\text { TPB } \\
175\end{array}$} & & TPA & TPA & TPA & TPD & TPD & TPD & TPD & TPD & TPB & TPB & TPB & & TPB & TPB & TPD & TPD \\
\hline & 345 & 347 & 208 & 020 & 024 & & & 185 & 009 & 007 & 014 & 110 & 115 & 172 & 168 & 169 & 243 & 246 & 236 & 338 & 335 & 240 & 095 & 099 \\
\hline$\overline{\mathrm{SiO}_{2}^{*}}$ & 55.03 & 51.08 & 50.99 & 48.01 & 53.33 & 53.81 & 52.03 & 51.08 & 56.38 & 59.25 & 65.84 & 73.96 & 74.30 & 73.93 & 69.10 & 68.11 & 69.31 & 69.33 & 67.92 & 62.87 & 69.68 & 69.08 & 72.31 & 70.30 \\
\hline $\mathrm{TiO}_{2}^{2}$ & 0.80 & 1.21 & 1.11 & 0.66 & 0.63 & 1.07 & 1.09 & 1.16 & 1.08 & 0.89 & 0.89 & 0.23 & 0.20 & 0.20 & 0.44 & 0.49 & 0.40 & 0.43 & 0.45 & 0.70 & 0.60 & 0.46 & 0.51 & 0.72 \\
\hline $\mathrm{Al}_{2} \mathrm{O}_{3}$ & 17.65 & 19.00 & 17.41 & 20.70 & 15.30 & 16.25 & 15.64 & 15.88 & 17.23 & 16.51 & 15.96 & 13.16 & 13.04 & 13.06 & 14.82 & 14.94 & 16.04 & 15.43 & 15.48 & 14.67 & 14.11 & 10.79 & 13.51 & 14.33 \\
\hline $\mathrm{Fe}_{2} \mathrm{O}_{3}$ & 5.67 & 6.54 & 8.34 & 6.46 & 8.70 & 7.55 & 8.02 & 9.41 & 7.84 & 6.71 & 5.64 & 2.25 & 2.16 & 2.29 & 2.78 & 3.21 & 2.51 & 3.05 & 3.01 & 5.42 & 4.55 & 6.78 & 3.55 & 4.96 \\
\hline $\mathrm{MnO}$ & 0.10 & 0.10 & 0.08 & 0.10 & 0.14 & 0.12 & 0.14 & 0.11 & 0.14 & 0.12 & 0.08 & 0.03 & 0.03 & 0.03 & 0.05 & 0.05 & 0.04 & 0.11 & 0.05 & 0.10 & 0.07 & 0.04 & 0.06 & 0.07 \\
\hline $\mathrm{MgO}$ & 6.41 & 6.55 & 6.47 & 11.46 & 7.41 & 6.21 & 9.43 & 7.74 & 4.50 & 4.27 & 2.18 & 0.34 & 0.27 & 0.20 & 1.61 & 1.83 & 1.78 & 1.54 & 1.96 & 2.42 & 1.70 & 1.64 & 1.30 & 1.85 \\
\hline $\mathrm{CaO}$ & 8.64 & 9.22 & 11.67 & 10.16 & 10.96 & 7.62 & 5.72 & 11.18 & 8.37 & 6.60 & 3.54 & 1.14 & 0.97 & 1.08 & 3.44 & 3.58 & 3.00 & 2.75 & 3.64 & 5.18 & 2.92 & 1.69 & 2.34 & 0.98 \\
\hline $\mathrm{Na}_{2} \mathrm{O}$ & 4.15 & 4.31 & 3.07 & 2.24 & 3.65 & 5.77 & 3.04 & 2.81 & 3.58 & 4.10 & 3.94 & 4.59 & 4.62 & 4.71 & 3.95 & 4.09 & 4.59 & 4.40 & 4.20 & 3.51 & 2.71 & 2.55 & 3.57 & 2.57 \\
\hline $\mathrm{K}_{2} \mathrm{O}$ & 0.52 & 0.38 & 0.10 & 0.04 & 0.04 & 0.37 & 1.16 & 0.52 & 0.52 & 1.05 & 2.09 & 3.12 & 3.26 & 3.11 & 2.28 & 2.05 & 1.51 & 1.75 & 2.06 & 1.55 & 2.33 & 1.19 & 2.05 & 2.42 \\
\hline $\mathrm{P}_{2}^{2} \mathrm{O}_{5}$ & 0.11 & 0.17 & 0.12 & 0.04 & 0.05 & 0.13 & 0.12 & 0.11 & 0.14 & 0.11 & 0.20 & 0.04 & 0.03 & 0.04 & 0.08 & 0.09 & 0.10 & 0.09 & 0.11 & 0.17 & 0.14 & 0.09 & 0.14 & 0.17 \\
\hline Total & 99.07 & 98.55 & 99.34 & 99.86 & 100.20 & 98.88 & 96.38 & 100.00 & 99.78 & 99.61 & 100.35 & 98.87 & 98.87 & 98.64 & 98.55 & 98.44 & 99.28 & 98.87 & 98.88 & 96.58 & $\mathbf{9 8 . 8 0}$ & 94.30 & 99.32 & 98.35 \\
\hline $\mathrm{Sc}^{* *}$ & 26 & 30 & 33 & 15 & 44 & 30 & 30 & 39 & 28 & 27 & 16 & 5 & 6 & 6 & 10 & 10 & 7 & 7 & 10 & 17 & 13 & 9 & 9 & 10 \\
\hline $\mathrm{V}$ & 143 & 196 & 203 & 108 & 209 & 194 & 188 & 223 & 186 & 162 & 128 & 15 & 9 & 11 & 51 & 54 & 43 & 44 & 59 & 119 & 97 & 67 & 68 & 86 \\
\hline Co & 38 & 37 & 49 & 64 & 72 & 42 & 40 & 66 & 61 & 60 & 74 & 107 & 112 & 102 & 84 & 76 & 73 & 69 & 79 & 44 & 44 & 88 & 61 & 37 \\
\hline $\mathrm{Zn}$ & 51 & 52 & 109 & 43 & 31 & 56 & 63 & 16 & 101 & 72 & 68 & 35 & 31 & 29 & 32 & 33 & 40 & 161 & 42 & 64 & 37 & 27 & 68 & 74 \\
\hline $\mathrm{Ga}$ & 16 & 16 & 15 & 13 & 16 & 15 & 15 & 15 & 18 & 16 & 20 & 16 & 15 & 15 & 15 & 16 & 18 & 18 & 18 & 18 & 17 & 14 & 17 & 19 \\
\hline $\mathrm{Rb}$ & 14 & 10 & 3.9 & 0.4 & 1.0 & 14 & 55 & 25 & 19 & 38 & 89 & 132 & 141 & 137 & 72 & 68 & 50 & 41 & 77 & 45 & 77 & 43 & 86 & 120 \\
\hline $\mathrm{Sr}$ & 228 & 239 & 238 & 125 & 122 & 170 & 295 & 142 & 235 & 271 & 212 & 55 & 51 & 53 & 169 & 169 & 268 & 188 & 271 & 166 & 220 & 174 & 337 & 112 \\
\hline Y & 17 & 25 & 25 & 12 & 25 & 26 & 26 & 25 & 22 & 29 & 29 & 36 & 37 & 39 & 20 & 23 & 13 & 19 & 17 & 23 & 26 & 17 & 28 & 32 \\
\hline $\mathrm{Zr}$ & 83 & 105 & 83 & 35 & 42 & 105 & 107 & 77 & 100 & 155 & 215 & 217 & 219 & 223 & 161 & 168 & 129 & 160 & 157 & 170 & 157 & 153 & 235 & 286 \\
\hline $\mathrm{Nb}$ & 4 & 6 & 4 & 0.8 & 0.6 & 5 & 5 & 3 & 6 & 7 & 12 & 10 & 8 & 9 & 6 & 7 & 4 & 7 & 8 & 7 & 9 & 8 & 11 & 16 \\
\hline Cs & 1.6 & 1.4 & 2.9 & 1.6 & 1.8 & 1.6 & 11.5 & 3.9 & 1.8 & 1.0 & 5.2 & 9.2 & 10.6 & 6.4 & 8.1 & 3.6 & 3.5 & 1.4 & 3.4 & 3.7 & 8.3 & 4.0 & 5.6 & 6.4 \\
\hline $\mathrm{Ba}$ & 95 & 90 & 41 & 10 & 16 & 66 & 109 & 60 & 151 & 229 & 424 & 475 & 470 & 459 & 525 & 465 & 285 & 489 & 377 & 204 & 479 & 203 & 441 & 422 \\
\hline $\mathrm{La}$ & 6.25 & 7.45 & 3.45 & 0.55 & 1.0 & 7.69 & 6.76 & 3.43 & 11.6 & 15.5 & 25.3 & 31.9 & 39.1 & 37.4 & 14.7 & 12.1 & 13.0 & 8.66 & 18.0 & 18.9 & 22.7 & 20.2 & 38.3 & 44.3 \\
\hline $\mathrm{Ce}$ & 13.4 & 17.1 & 9.16 & 2.68 & 3.8 & 16.9 & 17.6 & 9.76 & 23.4 & 34.8 & 48.9 & 71.3 & 84.6 & 71.5 & 34.0 & 27.8 & 31.0 & 20.1 & 37.7 & 41.1 & 46.0 & 40.6 & 80.4 & 89.5 \\
\hline $\operatorname{Pr}$ & 1.70 & 2.20 & 1.34 & 0.49 & 0.79 & 2.15 & 2.28 & 1.47 & 2.97 & 4.05 & 5.68 & 7.66 & 8.25 & 7.67 & 3.55 & 3.04 & 3.13 & 2.02 & 4.01 & 4.66 & 5.20 & 4.39 & 8.27 & 9.91 \\
\hline $\mathrm{Nd}$ & 7.48 & 10.1 & 6.83 & 2.74 & 4.9 & 11.0 & 10.2 & 8.06 & 13.3 & 17.5 & 24.5 & 28.1 & 29.4 & 28.2 & 13.6 & 12.7 & 12.1 & 7.51 & 14.8 & 18.8 & 19.7 & 17.3 & 30.2 & 35.0 \\
\hline $\mathrm{Sm}$ & 1.89 & 3.09 & 2.33 & 0.98 & 2.21 & 3.03 & 2.97 & 2.74 & 3.07 & 4.34 & 5.06 & 6.21 & 5.52 & 5.75 & 2.94 & 2.77 & 2.54 & 1.41 & 3.03 & 4.01 & 4.08 & 3.52 & 5.65 & 7.28 \\
\hline $\mathrm{Eu}$ & 0.95 & 1.25 & 0.89 & 0.67 & 0.94 & 1.09 & 1.06 & 1.14 & 1.38 & 1.25 & 1.30 & 0.68 & 0.77 & 0.78 & 0.66 & 0.67 & 0.77 & 0.34 & 1.00 & 1.05 & 0.90 & 0.88 & 1.10 & 1.55 \\
\hline $\mathrm{Gd}$ & 2.28 & 3.05 & 2.89 & 1.41 & 2.55 & 3.45 & 3.35 & 3.73 & 3.43 & 4.04 & 4.52 & 5.86 & 6.33 & 5.71 & 3.26 & 2.67 & 1.91 & 1.44 & 2.33 & 3.32 & 4.07 & 3.06 & 5.22 & 5.70 \\
\hline $\mathrm{Tb}$ & 0.41 & 0.56 & 0.50 & 0.23 & 0.50 & 0.55 & 0.62 & 0.60 & 0.58 & 0.65 & 0.81 & 0.98 & 0.99 & 0.95 & 0.44 & 0.46 & 0.30 & 0.26 & 0.39 & 0.56 & 0.59 & 0.42 & 0.74 & 0.97 \\
\hline Dy & 2.55 & 3.21 & 3.27 & 1.59 & 3.53 & 3.78 & 3.73 & 3.47 & 3.52 & 4.64 & 4.99 & 6.18 & 5.54 & 6.06 & 2.80 & 3.07 & 1.81 & 1.31 & 2.41 & 3.31 & 2.94 & 2.75 & 3.87 & 5.50 \\
\hline Но & 0.50 & 0.74 & 0.71 & 0.34 & 0.82 & 0.88 & 0.80 & 0.80 & 0.73 & 0.93 & 1.05 & 1.25 & 1.23 & 1.43 & 0.55 & 0.65 & 0.43 & 0.30 & 0.57 & 0.70 & 0.72 & 0.51 & 0.82 & 1.12 \\
\hline $\mathrm{Er}$ & 1.47 & 1.98 & 2.06 & 0.83 & 2.12 & 2.30 & 2.24 & 2.27 & 1.99 & 2.64 & 2.78 & 3.45 & 3.20 & 3.96 & 1.63 & 1.77 & 1.09 & 0.78 & 1.51 & 1.85 & 2.07 & 1.45 & 1.98 & 3.35 \\
\hline $\mathrm{Tm}$ & 0.22 & 0.27 & 0.30 & 0.14 & 0.33 & 0.36 & 0.32 & 0.33 & 0.32 & 0.39 & 0.41 & 0.55 & 0.41 & 0.65 & 0.27 & 0.29 & 0.17 & 0.12 & 0.23 & 0.29 & 0.30 & 0.24 & 0.32 & 0.39 \\
\hline $\mathrm{Yb}$ & 1.62 & 2.08 & 2.10 & 1.08 & 2.26 & 2.24 & 2.27 & 2.32 & 2.04 & 2.77 & 2.93 & 4.06 & 3.56 & 4.37 & 1.88 & 1.87 & 1.21 & 0.82 & 1.32 & 1.92 & 2.27 & 1.60 & 2.27 & 3.04 \\
\hline $\mathrm{Lu}$ & 0.21 & 0.29 & 0.28 & 0.16 & 0.30 & 0.32 & 0.34 & 0.33 & 0.28 & 0.40 & 0.47 & 0.56 & 0.50 & 0.66 & 0.25 & 0.35 & 0.15 & 0.14 & 0.21 & 0.31 & 0.43 & 0.25 & 0.32 & 0.46 \\
\hline $\mathrm{Hf}$ & 2.02 & 2.21 & 1.73 & 0.79 & 1.30 & 2.43 & 2.36 & 1.88 & 2.35 & 3.66 & 6.35 & 6.71 & 6.64 & 6.43 & 4.11 & 3.79 & 3.40 & 2.04 & 3.58 & 4.18 & 3.58 & 3.90 & 5.39 & 8.29 \\
\hline $\mathrm{Ta}$ & 0.24 & 0.28 & 0.21 & 0.03 & 0.04 & 0.25 & 0.26 & 0.15 & 0.34 & 0.43 & 0.76 & 0.74 & 0.81 & 0.81 & 0.44 & 0.46 & 0.45 & 0.25 & 0.57 & 0.43 & 0.61 & 0.53 & 0.70 & 0.99 \\
\hline $\mathrm{Pb}$ & 2.98 & 1.46 & 1.89 & n.d. & 0.26 & 2.19 & 2.11 & 0.37 & 17.5 & 5.10 & 15.7 & 11.2 & 13.6 & 12.1 & 12.4 & 10.7 & 10.8 & 9.17 & 10.7 & 10.3 & 7.34 & 6.35 & 18.5 & 17.7 \\
\hline Th & 1.01 & 0.90 & 0.53 & 0.01 & $<0.04$ & 1.61 & 1.37 & 0.51 & 3.53 & 4.74 & 8.41 & 11.9 & 11.3 & 15.4 & 7.28 & 5.94 & 5.39 & 3.81 & 6.56 & 6.17 & 7.00 & 5.94 & 12.3 & 13.1 \\
\hline U & 0.42 & 0.37 & 0.23 & 0.01 & $<0.01$ & 0.52 & 0.45 & 0.19 & 0.64 & 1.26 & 1.98 & 3.52 & 4.67 & 3.56 & 3.18 & 3.11 & 2.54 & 1.47 & 2.04 & 1.89 & 2.35 & 1.64 & 4.21 & 3.91 \\
\hline $\mathrm{Mg}^{* * * *}$ & 69.14 & 66.49 & 60.59 & 77.85 & 62.79 & 61.97 & 69.97 & 61.97 & 53.21 & 55.77 & 43.37 & 23.04 & 19.85 & 14.75 & 53.43 & 53.04 & 58.42 & 50.01 & 56.34 & 46.94 & 42.54 & 32.40 & 42.05 & 42.50 \\
\hline $\mathrm{ASI}^{* * * * *}$ & 0.76 & 0.78 & 0.66 & 0.93 & 0.59 & 0.68 & 0.94 & 0.62 & 0.80 & 0.83 & 1.05 & 1.01 & 1.01 & 1.00 & 0.97 & 0.97 & 1.10 & 1.09 & 0.98 & 0.87 & 1.15 & 1.26 & 1.09 & 1.66 \\
\hline $\mathrm{T}(\mathrm{Zr})$ & n.d. & n.d. & n.d. & n.d. & n.d. & n.d. & n.d. & n.d. & n.d. & n.d. & 801 & 811 & 813 & 814 & 774 & 775 & 767 & 786 & 770 & n.d. & n.d. & n.d. & n.d. & n.d. \\
\hline
\end{tabular}


Table 3. Sr and Nd isotopic ratios for the Taitao Ophiolite, the Taitao granites, sediments, and basement rocks

\begin{tabular}{|c|c|c|c|c|c|c|c|c|c|c|}
\hline \multicolumn{3}{|c|}{ Geological Unit } & Sample ID & ${ }^{87} \mathrm{Sr} /{ }^{86} \mathrm{Sr}$ & ${ }^{87} \mathrm{Rb} /{ }^{86} \mathrm{Sr}$ & ${ }^{87} \mathrm{Sr} /{ }^{86} \mathrm{Sr} i{ }^{*}$ & ${ }^{143} \mathrm{Nd} /{ }^{144} \mathrm{Nd}$ & ${ }^{147} \mathrm{Sm} /{ }^{144} \mathrm{Nd}$ & ${ }^{143} \mathrm{Nd} /{ }^{144} \mathrm{Nd} i$ & $\varepsilon N d i^{* *}$ \\
\hline \multirow{6}{*}{ 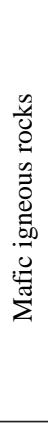 } & \multirow{4}{*}{ 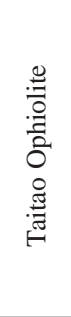 } & MVU & $\begin{array}{l}\text { TPB345 } \\
\text { TPB347 }\end{array}$ & $\begin{array}{l}0.703523( \pm 5) \\
0.703408( \pm 5)\end{array}$ & $\begin{array}{l}0.173 \\
0.127\end{array}$ & $\begin{array}{l}0.703510 \\
0.703399\end{array}$ & $\begin{array}{l}0.512957( \pm 4) \\
0.513038( \pm 4)\end{array}$ & $\begin{array}{l}0.1526 \\
0.1859\end{array}$ & $\begin{array}{l}0.512952 \\
0.513031\end{array}$ & $\begin{array}{l}6.25 \\
7.81\end{array}$ \\
\hline & & EC-SDC & TPD208 & $0.704236( \pm 5)$ & 0.047 & 0.704233 & $0.51306( \pm 3)$ & 0.2067 & 0.513053 & 8.22 \\
\hline & & Gabbro & $\begin{array}{l}\text { ТРВ020 } \\
\text { TPB024 }\end{array}$ & $\begin{array}{l}0.702808( \pm 5) \\
0.703175( \pm 5)\end{array}$ & $\begin{array}{l}0.009 \\
0.024\end{array}$ & $\begin{array}{l}0.702807 \\
0.703173\end{array}$ & $\begin{array}{l}0.513162( \pm 5) \\
0.513156( \pm 3)\end{array}$ & $\begin{array}{l}0.2168 \\
0.2699\end{array}$ & $\begin{array}{l}0.513154 \\
0.513146\end{array}$ & $\begin{array}{l}10.21 \\
10.05\end{array}$ \\
\hline & & $\mathrm{CMU}$ & $\begin{array}{l}\text { TPB174 } \\
\text { TPB175 }\end{array}$ & $\begin{array}{l}0.705274( \pm 6) \\
0.706713( \pm 4)\end{array}$ & $\begin{array}{l}0.230 \\
0.541\end{array}$ & $\begin{array}{l}0.705257 \\
0.706674\end{array}$ & $\begin{array}{l}0.512909( \pm 3) \\
0.512934( \pm 5)\end{array}$ & $\begin{array}{l}0.1661 \\
0.1754\end{array}$ & $\begin{array}{l}0.512903 \\
0.512928\end{array}$ & $\begin{array}{l}5.31 \\
5.79\end{array}$ \\
\hline & $\mathscr{0}$ & EC-dike & TPD185 & $0.703181( \pm 4)$ & 0.503 & 0.703145 & $0.513119( \pm 4)$ & 0.2054 & 0.513112 & 9.38 \\
\hline & & TM-dike & TPA009 & $0.707171( \pm 5)$ & 0.235 & 0.707154 & $0.512611( \pm 5)$ & 0.1400 & 0.512606 & -0.49 \\
\hline \multirow{5}{*}{ 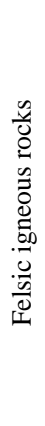 } & \multirow{5}{*}{ 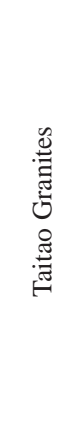 } & $\mathrm{TM}$ & $\begin{array}{l}\text { TPA007 } \\
\text { TPA014 }\end{array}$ & $\begin{array}{l}0.708212( \pm 3) \\
0.707458( \pm 5)\end{array}$ & $\begin{array}{l}0.402 \\
1.214\end{array}$ & $\begin{array}{l}0.708179 \\
0.707360\end{array}$ & $\begin{array}{c}0.512448( \pm 3) \\
0.512705( \pm 19)\end{array}$ & $\begin{array}{l}0.1500 \\
0.1251\end{array}$ & $\begin{array}{l}0.512443 \\
0.512701\end{array}$ & $\begin{array}{l}-3.68 \\
1.36\end{array}$ \\
\hline & & SH & $\begin{array}{l}\text { TPD110 } \\
\text { TPD115 } \\
\text { TPD172 } \\
\end{array}$ & $\begin{array}{l}0.704587( \pm 4) \\
0.704795( \pm 3) \\
0.704821( \pm 3) \\
\end{array}$ & $\begin{array}{l}6.992 \\
7.998 \\
7.493 \\
\end{array}$ & $\begin{array}{l}0.704081 \\
0.704216 \\
0.704278 \\
\end{array}$ & $\begin{array}{l}0.512839( \pm 3) \\
0.512828( \pm 3) \\
0.512797( \pm 5)\end{array}$ & $\begin{array}{l}0.1339 \\
0.1136 \\
0.1233 \\
\end{array}$ & $\begin{array}{l}0.512834 \\
0.512824 \\
0.512793 \\
\end{array}$ & $\begin{array}{l}3.96 \\
3.76 \\
3.15 \\
\end{array}$ \\
\hline & & $\mathrm{EC}$ & $\begin{array}{l}\text { TPD168 } \\
\text { TPD169 } \\
\end{array}$ & $\begin{array}{l}0.705430( \pm 3) \\
0.705440( \pm 3)\end{array}$ & $\begin{array}{l}1.242 \\
1.164 \\
\end{array}$ & $\begin{array}{l}0.705340 \\
0.705356 \\
\end{array}$ & $\begin{array}{l}0.512661( \pm 4) \\
0.512676( \pm 6)\end{array}$ & $\begin{array}{l}0.1305 \\
0.1323 \\
\end{array}$ & $\begin{array}{l}0.512656 \\
0.512671 \\
\end{array}$ & $\begin{array}{l}0.49 \\
0.79\end{array}$ \\
\hline & & BB & $\begin{array}{l}\text { TPB243 } \\
\text { TPB246 } \\
\end{array}$ & $\begin{array}{l}0.705155( \pm 3) \\
0.705404( \pm 4) \\
\end{array}$ & $\begin{array}{l}0.542 \\
0.630\end{array}$ & $\begin{array}{l}0.705117 \\
0.705360 \\
\end{array}$ & $\begin{array}{l}0.512756( \pm 4) \\
0.512667( \pm 9)\end{array}$ & $\begin{array}{l}0.1273 \\
0.1135 \\
\end{array}$ & $\begin{array}{l}0.512751 \\
0.512663 \\
\end{array}$ & $\begin{array}{l}2.35 \\
0.62 \\
\end{array}$ \\
\hline & & $\mathrm{CR}$ & TPB236 & $0.704972( \pm 5)$ & 0.820 & 0.704927 & $0.512714( \pm 4)$ & 0.1238 & 0.512710 & 1.53 \\
\hline \multirow{2}{*}{ 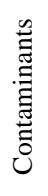 } & \multicolumn{2}{|c|}{ Sediment } & TPB338 & $0.706549( \pm 3)$ & 0.781 & 0.706495 & $0.512102( \pm 3)$ & 0.1291 & 0.512097 & -10.41 \\
\hline & \multicolumn{2}{|c|}{ Basement } & $\begin{array}{l}\text { TPB240 } \\
\text { TPB335 } \\
\text { TPD095 } \\
\text { TPD099 }\end{array}$ & $\begin{array}{l}0.707882( \pm 5) \\
0.707408( \pm 5) \\
0.713521( \pm 5) \\
0.720999( \pm 5)\end{array}$ & $\begin{array}{l}0.723 \\
1.014 \\
0.741 \\
3.103\end{array}$ & $\begin{array}{l}0.707830 \\
0.707335 \\
0.713467 \\
0.720774\end{array}$ & $\begin{array}{l}0.512436( \pm 4) \\
0.512469( \pm 4) \\
0.512175( \pm 3) \\
0.512186( \pm 3)\end{array}$ & $\begin{array}{l}0.1235 \\
0.1250 \\
0.1129 \\
0.1260\end{array}$ & $\begin{array}{l}0.512432 \\
0.512465 \\
0.512171 \\
0.512181\end{array}$ & $\begin{array}{l}-3.89 \\
-3.25 \\
-8.97 \\
-8.77\end{array}$ \\
\hline
\end{tabular}

* Initial ratios (i) for the Sr and Nd isotopic ratios are calculated with the zircon age data from Anma et al., (2006, 2009), ** $\varepsilon N d i$ : deviation in parts per $10^{4}$ from bulk earth values at their initial age (zircon age). Basement rocks are calculated with $5.1 \mathrm{Ma}$ for comparison

(Figs. 4a and 5a). The gabbros had lower $\mathrm{TiO}_{2}, \mathrm{~K}_{2} \mathrm{O}$ and $\mathrm{P}_{2} \mathrm{O}_{5}$ contents (Fig. 3) than other mafic rocks and had N-MORB signatures, being depleted in large ion lithophile elements (LILEs) such as $\mathrm{Rb}$, Th and U (Fig. 4a). The other mafic rocks were relatively enriched in LILEs and slightly depleted in $\mathrm{Ba}, \mathrm{Nb}$ and $\mathrm{Ta}$. The mafic dike sample from the Tres Montes pluton (TM-dike in the figures and TPA009 in the tables) showed notable enrichment in $\mathrm{Pb}$ and $\mathrm{Th}$, possibly due to the contamination of crustal rocks (Fig. 4a). Gabbros (Fig. 5a) had depleted light REE (LREE) contents with a positive Eu anomaly that implies accumulation of Ca-plagioclase. MVU samples and the TMdike had less distinct positive Eu anomalies. Together with CMU samples, they had slightly LREE-enriched features. In the Estero Cono pluton, the samples from an isolated mafic dike (EC-dike; TPD185) and the sheeted dike complex (EC-SDC; TPD208) had a rather flat REE pattern. However, EC-SDC was slightly depleted in Rb, Ba and $\mathrm{K}$ and enriched in $\mathrm{Pb}$ compared to EC-dike, most likely due to alteration by the intrusion of the Seno Hoppner-Estero Cono pluton (Fig. 4a).

The gabbros and the EC-dike had the most primitive isotopic ratios among the mafic rocks we analyzed, ranging from 0.70281 to 0.70715 for ${ }^{87} \mathrm{Sr} /{ }^{86} \mathrm{Sr} i$ and from 10.21 to -0.49 for åNd $i$ values (Figs. 7 and 8). Compared to the gabbros, the MVU samples had slightly lower Nd isotopic ratios, whereas EC-SDC had a slightly higher ${ }^{87} \mathrm{Sr} /$ ${ }^{86} \mathrm{Sri}$. The samples from CMU and TM-dike had higher $\mathrm{Sr}$ and lower $\mathrm{Nd}$ isotopic values than the other mafic rocks.

The Taitao granites plot in three distinct fields, except for one hybrid rock with a tonalitic composition (Fig. 2b). Each pluton has relatively homogeneous major-element compositions. The samples from the Seno Hoppner pluton plot in the field for true granite (Fig. 2b) and coincide with the hornblende-free granitoids of the Seno Hoppner-Estero Cono pluton reported by Kon et al. (2013) (Fig. 4b, 5b). They have the highest silica contents among the Taitao granites (Fig. 3) and low $\mathrm{MgO}$ and $\mathrm{CaO}$ contents with low $\mathrm{Na}_{2} \mathrm{O} /$ $\mathrm{K}_{2} \mathrm{O}$ and $\mathrm{CaO} / \mathrm{Na}_{2} \mathrm{O}$ ratios (1.4-1.5 and 0.2, respectively) (Table 2). The samples from the Estero Cono and Cabo Raper plutons plot on the boundary between the tonalite and granodiorite fields, coinciding with the hornblende-bearing granitoids of the Seno Hoppner-Estero Cono and Cabo Raper plutons reported by Kon et al. (2013) (Fig. 4b, $5 b)$. The Tres Montes granodiorite plot in the same position as the hornblende-bearing granitoids (Fig. 2b), but has lower silica contents (Fig. 3). The samples from the Bahia Barrientos pluton have distinctive compositions, plotting on the boundary between the tonalite and trondhjemite fields (Fig. 2b). These samples have lower $\mathrm{K}_{2} \mathrm{O}$ contents and higher aluminum saturation index (ASI) values (Table 2) than other granites.

In other respects, the Estero Cono, Cabo Raper and Bahia Barrientos plutons have similar major-element compositions with slight variations in $\mathrm{Na}_{2} \mathrm{O}$ and $\mathrm{K}_{2} \mathrm{O}$ contents. The $\mathrm{Na}_{2} \mathrm{O} / \mathrm{K}_{2} \mathrm{O}$ ratios range from 1.7 to 3.0 , and the $\mathrm{CaO} / \mathrm{Na}_{2} \mathrm{O}$ ratios range from 0.6 to 0.9 . The ASI values (Table 2) indicate that the granites are metaluminous to slightly peraluminous and are classified as I-type granite (Chappell and White, 1974). All of the Taitao granites fall into the medium-K classification (Fig. 3). On the Sr/Y vs. Y diagram, all Taitao granites fell within the non-adakitic field (Fig. 6a). The Taitao granites range 
(a)

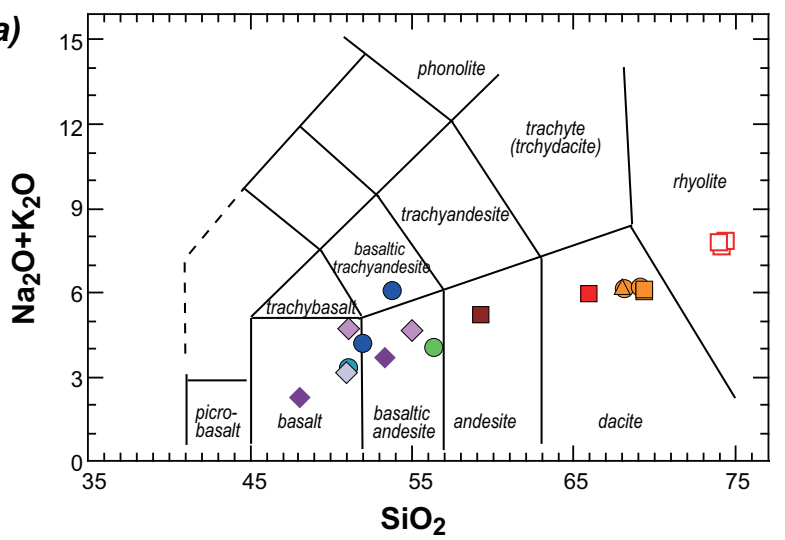

(b)

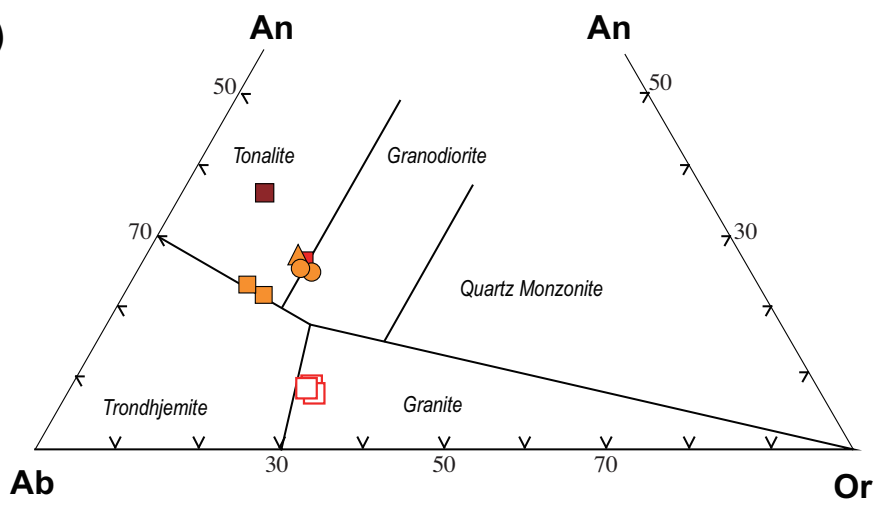

The Taitao Ophiolite and other mafic rocks $\diamond \mathrm{MVU} O \mathrm{CMU}$

$\checkmark$ EC-SDC $\bigcirc$ EC-dike

Gabbro TM-dike
The Taitao Granites

$\square \mathrm{SH} \square \mathrm{BB} \square \mathrm{TM}$

$\triangle \mathrm{CR} \square$ TM-hybrid

Figure 2. Chemical classification of the Taitao ophiolite and Taitao granites. (a) Total alkali vs. silica diagram after Le Maitre et al. (2005). (b) Normative anorthite (An)-albite (Ab)-orthoclase (Or) ternary diagram after Barker (1979).
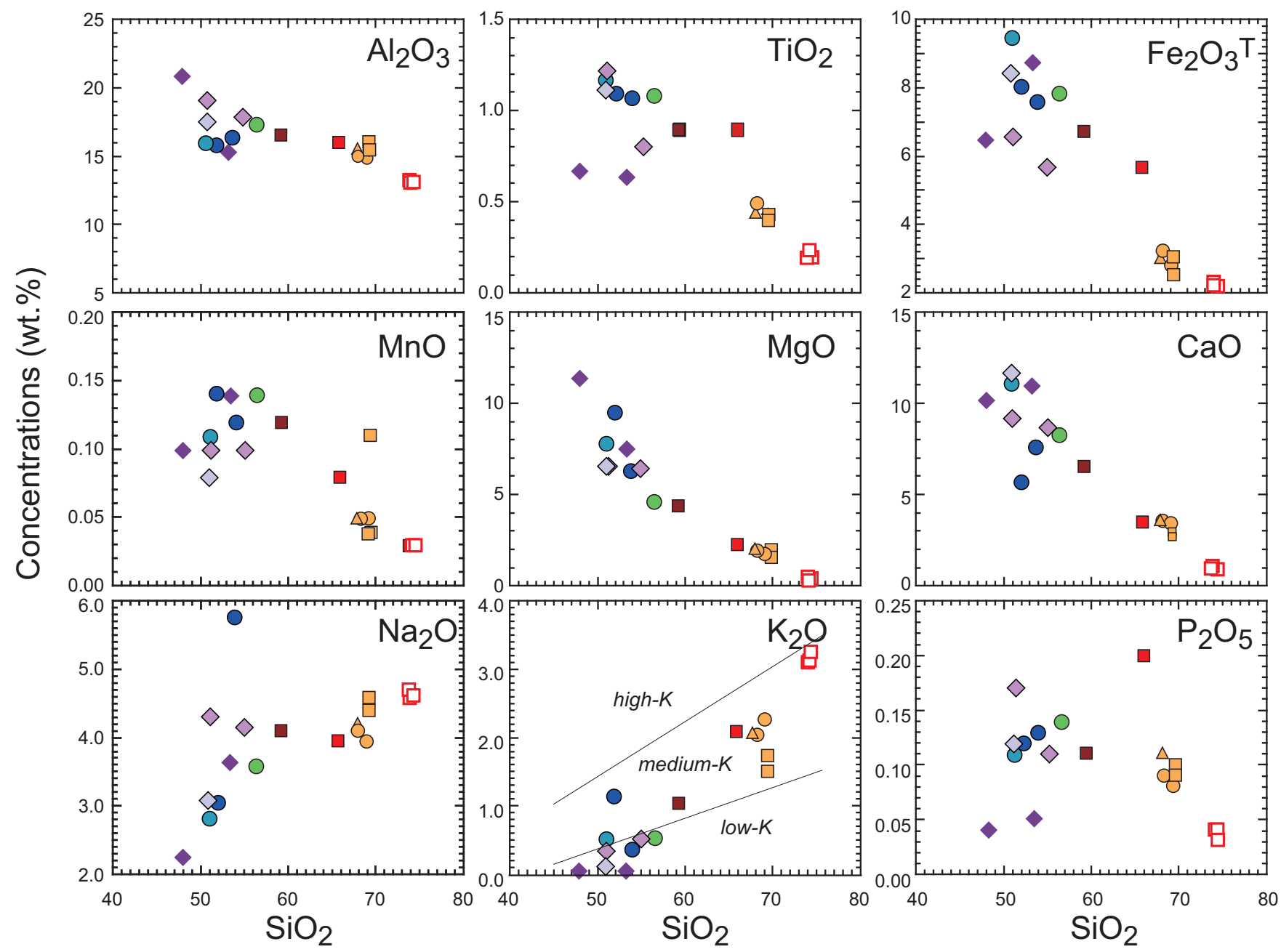

Figure 3. Harker variation diagrams for the Taitao ophiolite and Taitao granites. Symbols are the same as in Fig. 2. 

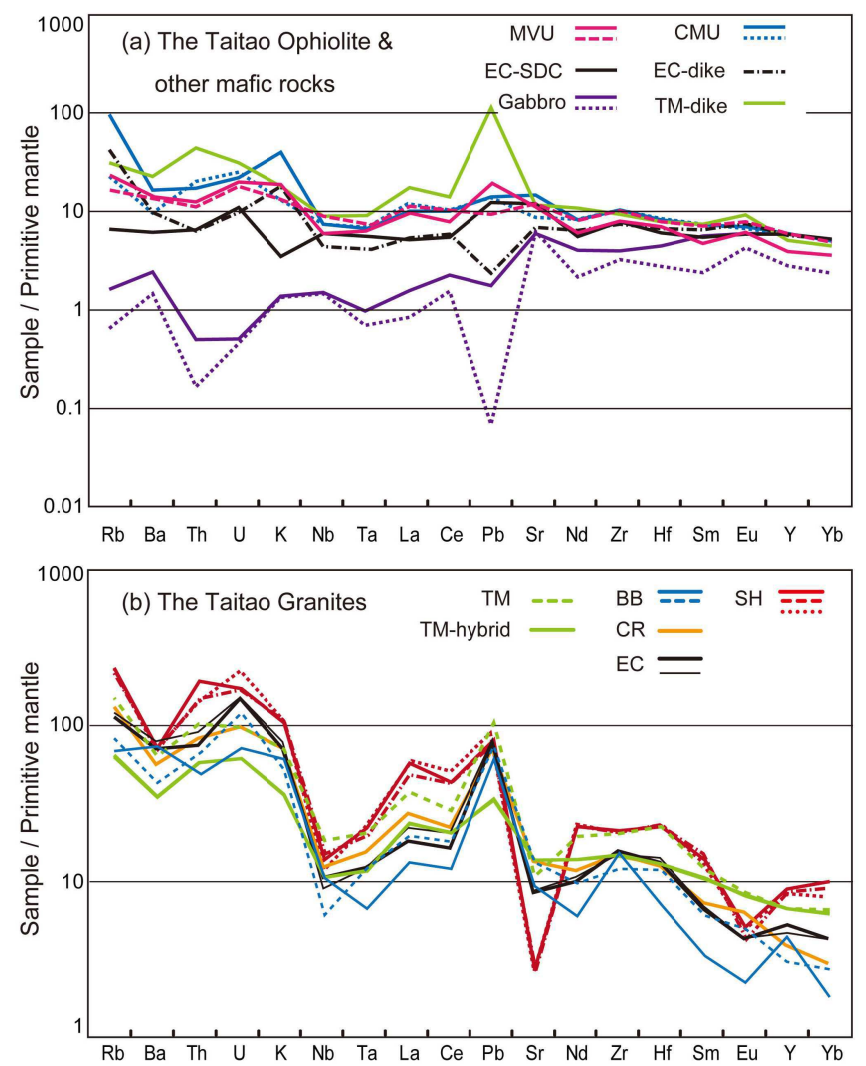

Figure 4. Trace-element variation diagrams for (a) the Taitao ophiolite and $(b)$ the Taitao granites normalized with respect to primitive mantle (McDonough and Sun, 1995).

in $\mathrm{Y}$ content from $17 \mathrm{ppm}$ to $39 \mathrm{ppm}$, but the Seno Hoppner pluton has distinctly high $\mathrm{Y}$ contents among them.

In their trace-element profiles, the Taitao granites appear enriched in LILEs and $\mathrm{Pb}$ with respect to primitive mantle (Fig. 4b). The granites are depleted in $\mathrm{Ba}, \mathrm{Nb}, \mathrm{Sr}$ and Eu and showed wide variation in REEs. The Seno Hoppner samples are distinguished by higher contents of most trace elements and greater depletions in $\mathrm{Sr}$ and Eu. The Tres Montes samples do not show negative anomalies of $\mathrm{Sr}$ and Eu. Sample TPB246 from the Bahia Barrientos granite differs from the others in its enrichment in $\mathrm{Ba}, \mathrm{Zr}$ and $\mathrm{Y}$ and depletion in Th, Ta and REEs. The chondrite-normalized REE patterns (Fig. 5 b) confirm these geochemical features, showing relatively enriched and inclined LREE and flattened HREE patterns. The Eu anomaly is large in the Seno Hoppner pluton, but slight or absent in the other granites.

The Taitao granites also fall into three groups with restricted $\mathrm{Sr}$ and $\mathrm{Nd}$ isotopic values (Figs. 7 and 8). The Seno Hoppner granite, with the highest $\mathrm{SiO}_{2}$ content of all felsic rocks in this area, has the most primitive isotopic composition: three ${ }^{87} \mathrm{Sr} /{ }^{86} \mathrm{Sr} i$ values range from 0.7045 to 0.7048 , and $\varepsilon N d i$ values range from 3.15 to 3.96 . The Tres Montes granodiorite, with the lowest $\mathrm{SiO}_{2}$ content among the Taitao granites, has the highest ${ }^{87} \mathrm{Sr} /{ }^{86} \mathrm{Sr} i$ value $(0.7073)$ and a relatively low $\varepsilon N d i$ value (1.36). Other plutons has intermediate ${ }^{87} \mathrm{Sr} /{ }^{86} \mathrm{Sr} i$ values $(0.7049-0.7053)$ and $\varepsilon N d i$ values ranging from 0.49 to 2.35. The hybrid granite in the Tres Montes pluton has an intermediate composition between those of the mafic dike and the host granodiorite; however, it has the highest ${ }^{87} \mathrm{Sr} /{ }^{86} \mathrm{Sri}(0.7073)$ and the lowest $\varepsilon N d i$ value $(-3.68)$ among the Taitao granites.
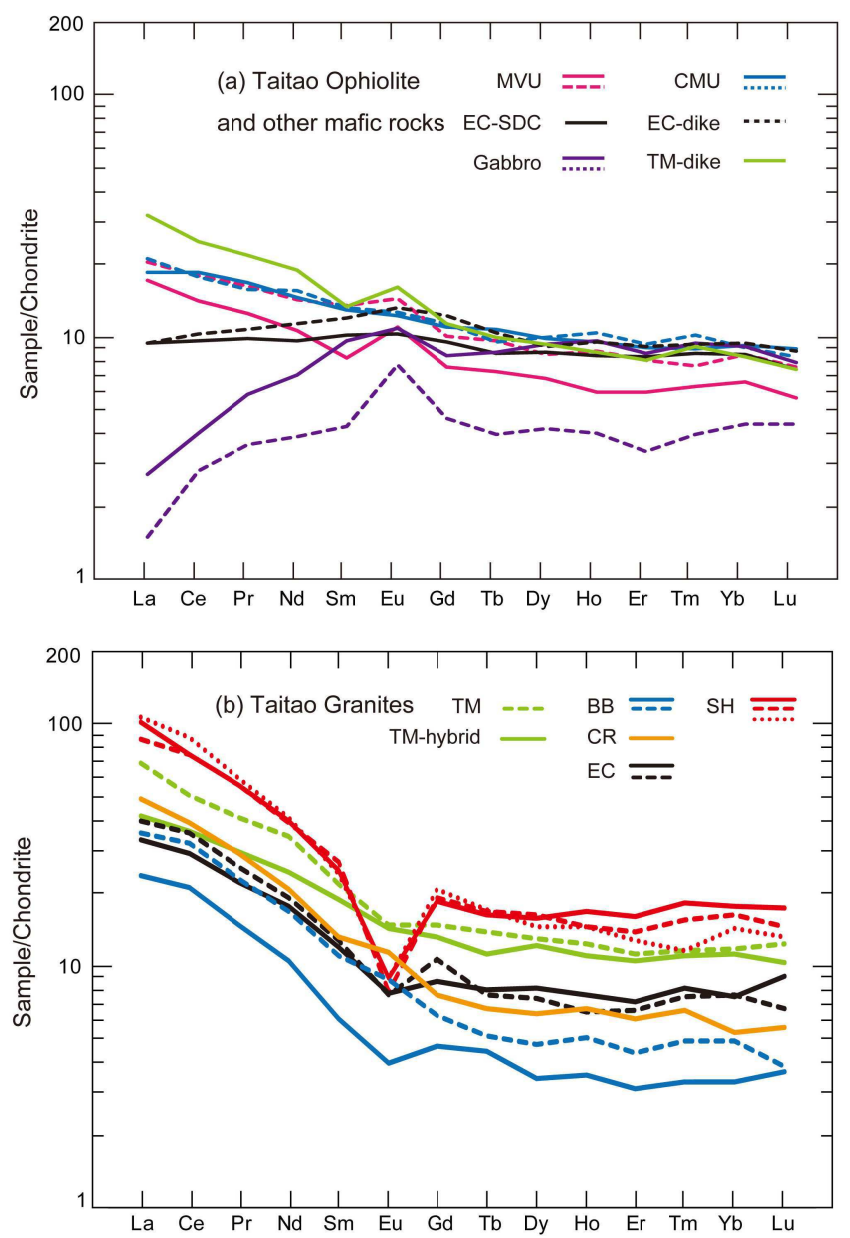

Figure 5. REE variation diagrams for (a) the Taitao ophiolite and (b) the Taitao granites normalized with respect to chondritic values (Taylor and McLennan, 1985).

The sediments in the MVU has the lowest Nd isotopic ratios $\left(-10.41\right.$ for $\left.\varepsilon \mathrm{Nd}_{5.5}\right)$ of all our samples. The basement rocks were divided into two groups by their $\varepsilon \mathrm{Nd}_{5.5}$ values, one with values around -3 and the other with values around -9 . The latter group of basement rocks has the highest ${ }^{87} \mathrm{Sr} /{ }^{86} \mathrm{Sri}$ values of our samples.

\section{Magmatic interaction in the ridge-trench intersection environment}

Our data indicate that the compositions of igneous rocks in the Taitao Peninsula area vary chemically and isotopically. Schulte et al. (2009) have demonstrated that gabbros and ultramafic rocks, which display rather restricted isotopic compositions in our data, have wider isotopic variations outside our study area ("reference data" in Fig. 8): most ultramafic rocks have ${ }^{87} \mathrm{Sr} /{ }^{86} \mathrm{Sr} i$ values higher than those of N-MORB gabbros. The highest ${ }^{87} \mathrm{Sr} /{ }^{86} \mathrm{Sr} i$ value was 0.708 , and six of our eight ultramafic samples had high $\varepsilon N d i$ values (9-10). Few gabbro samples plotted in that field (solid circles with $\varepsilon \mathrm{Nd} i>9$ in Fig. 8; Schulte et al., 2009). This trend implies alteration by seawater (indicated by arrow labeled "seawater alteration" in Fig. 8) with a ${ }^{87} \mathrm{Sr} /{ }^{86} \mathrm{Sr}$ value around 0.709 .

Our data from the MVU basalts have lower $\varepsilon N d i$ and slightly higher ${ }^{87} \mathrm{Sr} /{ }^{86} \mathrm{Sr} i$ than the gabbros and the EC-dike with N-MORB 

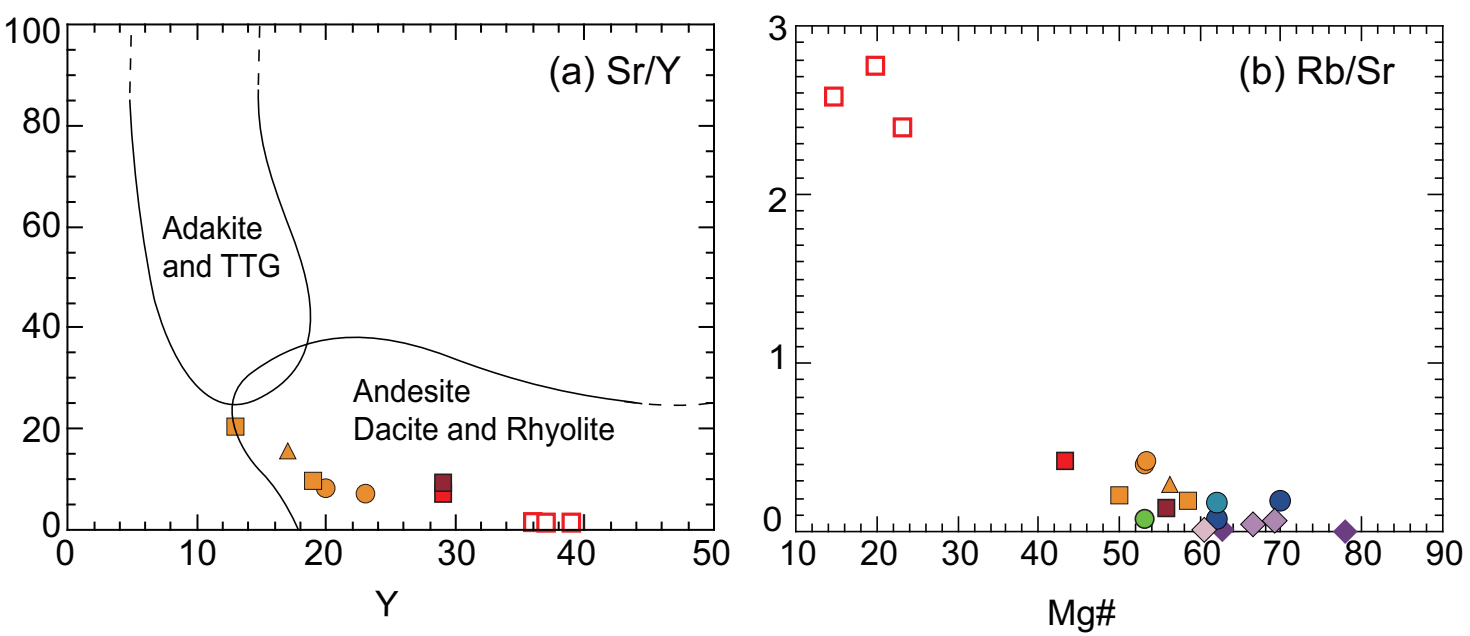

Figure 6. (a) Y vs. Sr/Y ratio diagram for the Taitao granites, and (b) Mg\# vs. Rb/Sr plot for all analyzed rocks.
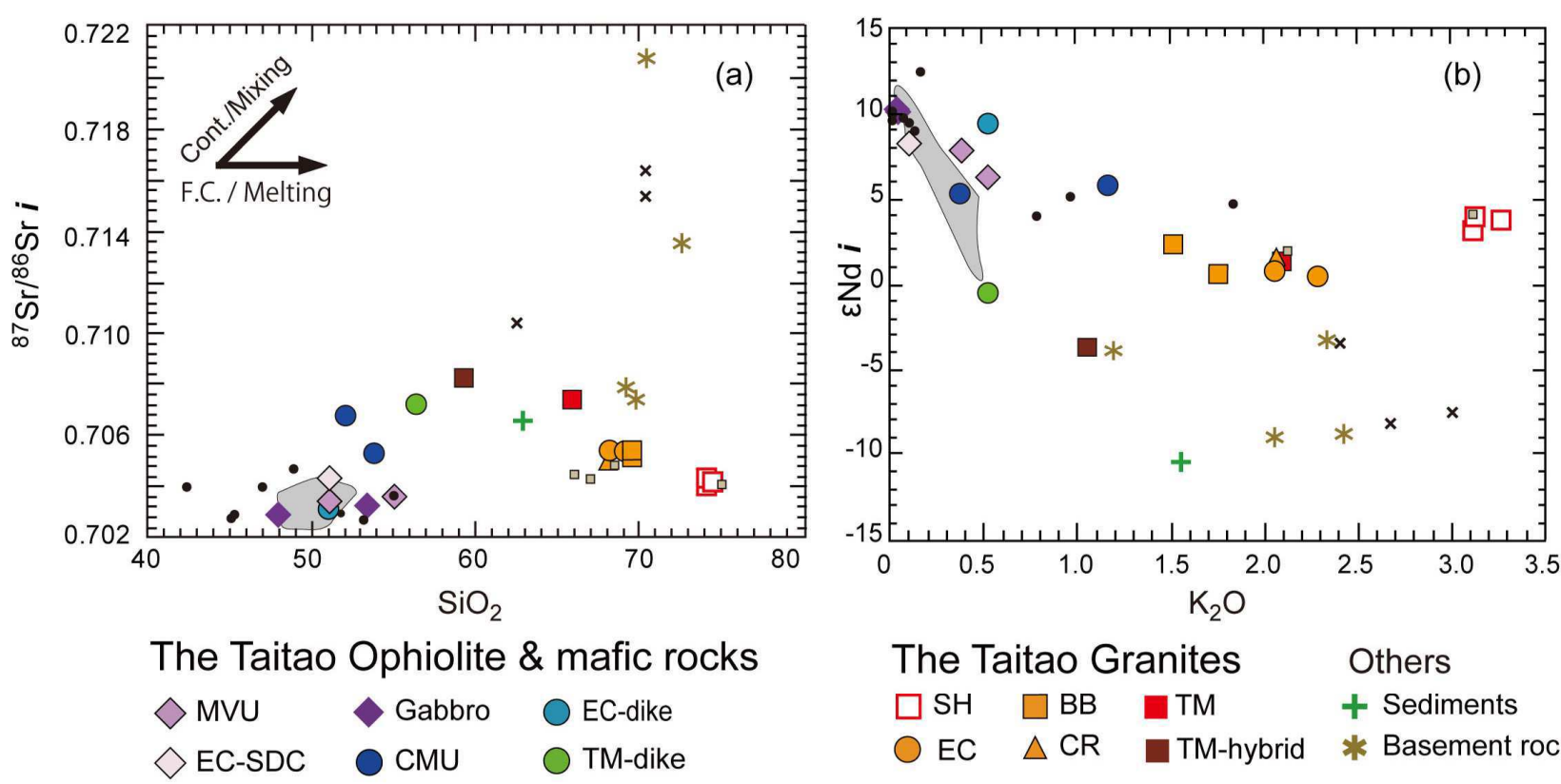

Others

$\begin{array}{lll}\square \mathrm{SH} & \square \mathrm{BB} & \square \mathrm{TM} \\ \mathrm{EC} & \triangle \mathrm{CR} & \square \mathrm{TM} \text {-hybrid }\end{array}$

+ Sediments

Reference data

- Chile ridge basalts
- Taitao ophiolite mafic rocks

$\square \quad$ Taitao granitic rocks
$\times$ Basement rocks

Figure 7. (a) $\mathrm{SiO}_{2}$ vs. ${ }^{87} \mathrm{Sr} /{ }^{86} \mathrm{Sri}$ diagram and (b) $\mathrm{K}_{2} \mathrm{O}$ vs. ENdi diagram for the Taitao ophiolite, Taitao granites, and basement rocks. Reference data sources: Klein and Karsten (1995) and Strum and Klein (1999) for Chile Ridge basalts, Kaeding et al. (1990) and Schulte et al. (2009) for Taitao ophiolite and granitic rocks.

composition. Some volcanic rocks from the Taitao ophiolite have been reported to have similar compositions (Kaeding et al., 1990; solid circles with $\varepsilon N d i=4-5$ in Fig. 8). The distribution of these rocks nearly coincides with that of the basalts dredged from the Chile ridge segments (gray field in Fig. 8; Klein and Karsten, 1995; Strum et al., 1999). Thus, these rocks were likely formed in a segment of the Chile ridge that had already subducted (segment -2 in Fig. 9a).

The volcanic rocks from the Chile ridge and from the Taitao ophiolite both plotted in a wider field that extends to lower $\varepsilon \mathrm{Nd} i$ values than the N-MORB field (Fig. 8). This isotopic variation implies that the source material of these rocks in the upper mantle was contaminated by crustal material with lower $\varepsilon N d$ value. The most probable candidate for such material is lower crust that has experienced partial melting (indicated by arrow labeled "deep crustal contamination" in Fig. 8). To introduce such material into the subridge area, Strum et al. (2000) suggested a mechanism in which suboceanic lithospheric mantle material foundered during subduction and was brought beneath the spreading ridge entering the subduction zone by mantle upwelling (Fig. 10a), or one in which old continental crust or metasomatized mantle beneath the arc communicated with mantle beneath the ridge through a slab window (Fig. 10b). The contaminant must have had an isotopic composition similar to the sediment in the MVU (TPB338; green cross in Fig. 8). However, this sample contained clastic zircons of the same age as the Taitao granites, and there is no rational explanation for the extremely low $\varepsilon \mathrm{Nd}$ value of this sediment. 


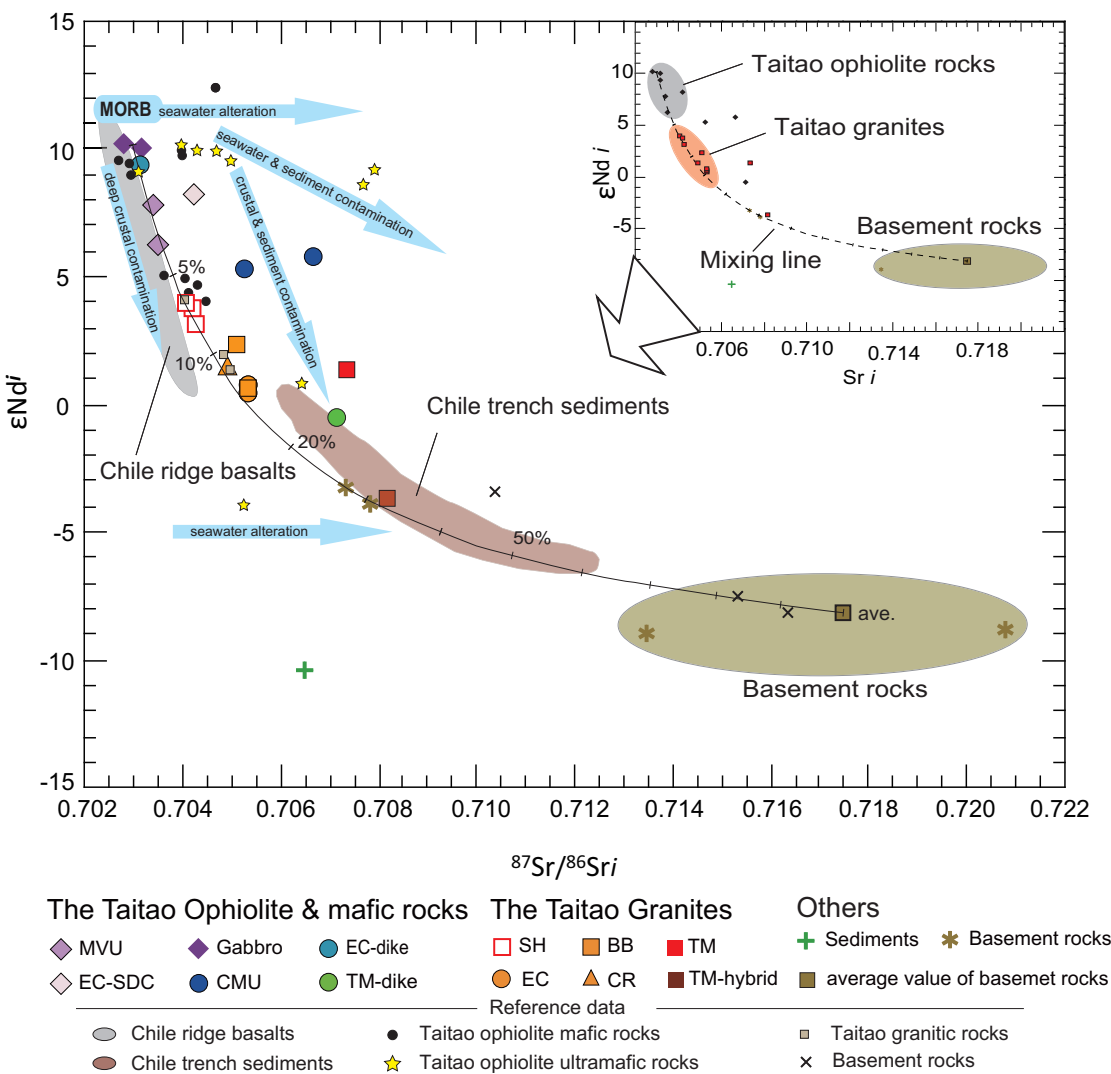

Figure $8 .{ }^{87} \mathrm{Sr} /{ }^{86}$ Sri vs. ENdi diagram for the Taitao ophiolite, Taitao granites, and basement rocks and sediments. The thin solid line is a mixing line that ties isotopic composition of average gabbro and average basement rocks. Reference data sources: Kilian and Behrmann (2003) for the Chile trench sediments, Klein and Karsten (1995) and Strum and Klein (1999) for Chile Ridge basalts, Kaeding et al. (1990) and Schulte et al. (2009) for Taitao ophiolite and granitic rocks. CHUR(0) $=0.512638$ (Wasserburg et al., 1981) was used for the calculation of $\varepsilon N d i$.

Alteration of the source mantle by deep crustal material and seawater must have been proceeding when segments of the Chile ridge were entering the subduction zone. The ultramafic section of the ophiolite underwent severe alteration by seawater, but no basaltic rocks from the Chile ridge and only a few gabbros of the Taitao ophiolite exhibited such evidence. In contrast, evidence for contamination of the sub-ridge mantle by deep crustal material has been observed in both the ridge and the ophiolite. Thus, pervasive contamination by deep crustal material must have been taking place before seawater alteration started as the ridge approached the subduction zone.

Anma et al. (2013) have shown that the magmatism that formed the 4.6 Ma CMU started in the South Taitao Ridge (Fig. 1b) at around 5.3 Ma and migrated eastward. They attributed this phenomenon to the subduction of the Tres Montes fracture zone. They also have reported evidence for the incorporation of trench-fill sediment that was brought to the upper mantle with the subducting fracture zone, then returned to the surface by volcanic activities of the CMU. Our new isotopic data support this model in that the mafic volcanic rocks of the CMU and TM-dike have higher ${ }^{87} \mathrm{Sr} /{ }^{86} \mathrm{Sr} i$ values than the Chile ridge basalts. The isotopic composition of the $\mathrm{CMU}$ may be explained in terms of marine alteration of basaltic rocks that were contaminated by deep crustal material (trajectory 1 in Fig. 8), contamination by trench-fill sediment of mantle that was altered by seawater (trajectory
3 in Fig. 8), or the combined influence of seawater alteration and sediment contamination of N-MORB magmas (trajectory 2 in Fig. 8). Alteration of mantle by seawater was most likely to occur along a fracture zone (Fig. 9a-d). As the fracture zone approached the subduction zone, sediment from the forearc area was carried onto it through submarine canyons. This fracture-filling sediment, accompanied by seawater, may have penetrated the crust during shear along the fracture zone and altered the mantle (Fig. 10c). The influence of slab tearing that resulted from buoyancy differences between the subducting slab segments may have enhanced this penetration.

\section{Origin of calc-alkaline I-type granites}

The fact that the Taitao granites are restricted to the vicinity of the Taitao ophiolite implies that the plutonism was related to the subduction of the hot ridge. The Taitao granites are calc-alkaline, meta-aluminous to slightly peraluminous $(0.83<$ ASI < 1.1), I-type granites. Such granites are generally considered to form by partial melting of an igneous source material. In the Taitao Peninsula region, possible source materials are limited to the subducting oceanic crust or igneous crustal material in the forearc region, if any. The low Sri ratios and high $\varepsilon N d$ ratios support the oceanic crustal origin of the granitic melts. However, contamination by sediment or other continental crustal material did occur, especially in the Tres Montes granodiorite, which contains over $40 \%$ recycled zircon of Cretaceous to Late Proterozoic ages (Anma et al., 2009)

The mixing line in Figure 8 represents a petrogenetic model based on $\mathrm{Sr}-\mathrm{Nd}$ isotopic compositions. Most Taitao granites plot on the mixing line between the average composition of gabbros, with primitive N-MORB isotopic compositions, and the average composition of metasedimentary basement rocks with the lowest $\varepsilon \mathrm{Nd}$ values, chosen as an extreme end-member. Taking N-MORB as the source material of the granite melts, our calculations indicate that the Seno Hoppner granite (with the most depleted isotopic composition) requires incorporation of no more than 5-10\% of contaminant. Other plutons, except the Tres Montes pluton, would require slightly more contamination to yield a result concordant with the calculation by Kaeding et al. (1990). Because recycled zircon is rare in these plutons, the contaminant could also be lower crustal material originating from a foundering slab or from sub-arc mantle that had experienced partial melting (Strum et al. 2000). Conversely, the major- and trace-element compositions of our samples favor contamination by sediment or basement of the upper crust. To produce the Tres Montes granodiorite requires a significant alteration by seawater or incorporation of sediment, most probably related to the subduction of the Esmeralda fracture zone (Fig. 9b, c).

The Taitao ophiolite has undergone low-pressure metamorphism at zeolite to amphibolite facies conditions (Shibuya et al., 2007). Experimental studies at less than $1 \mathrm{GPa}$ suggest that decomposition of amphiboles in hot oceanic crust could result in K-poor partial melts 


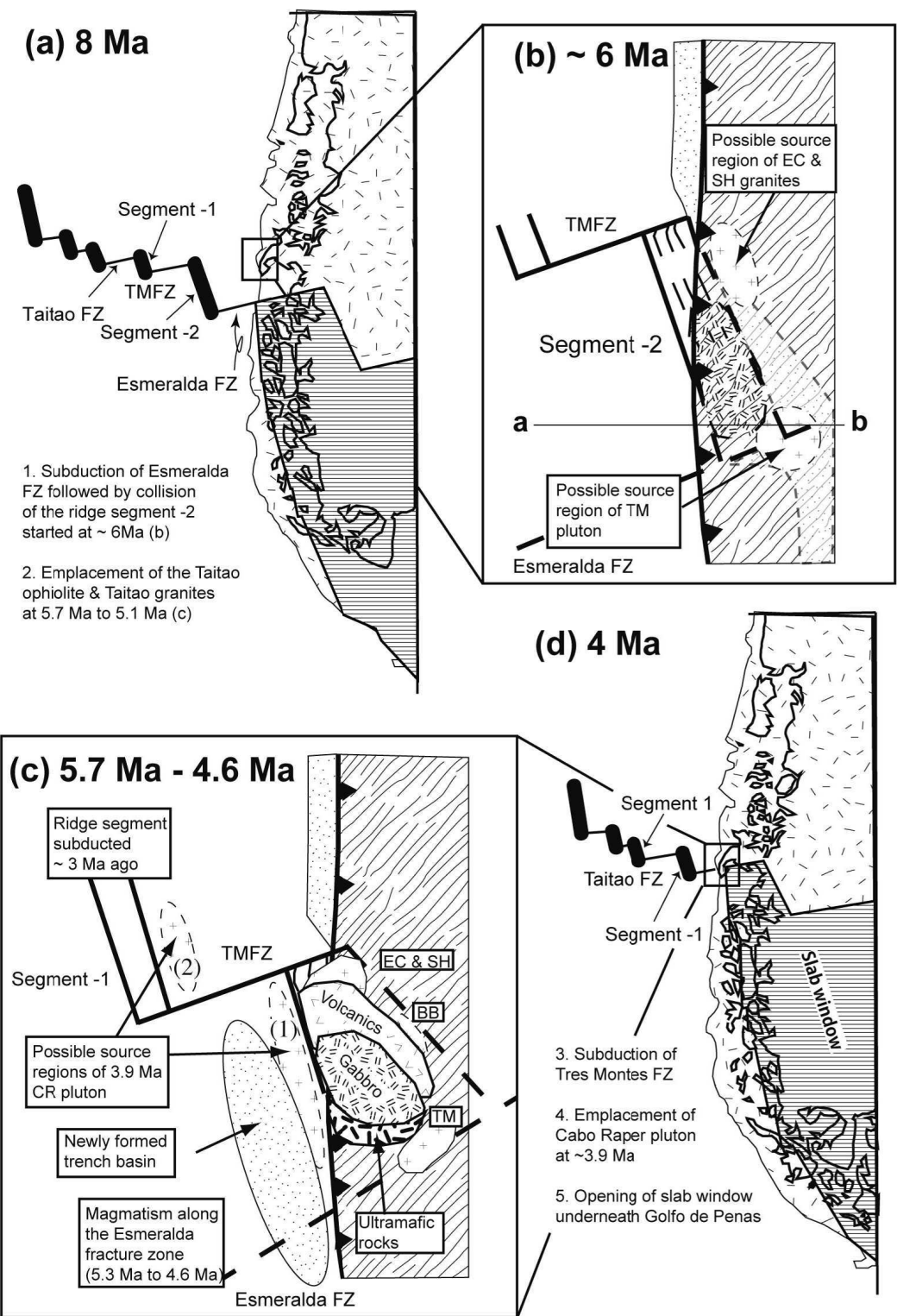

E) cross section (5.7 Ma - 5.1 Ma)

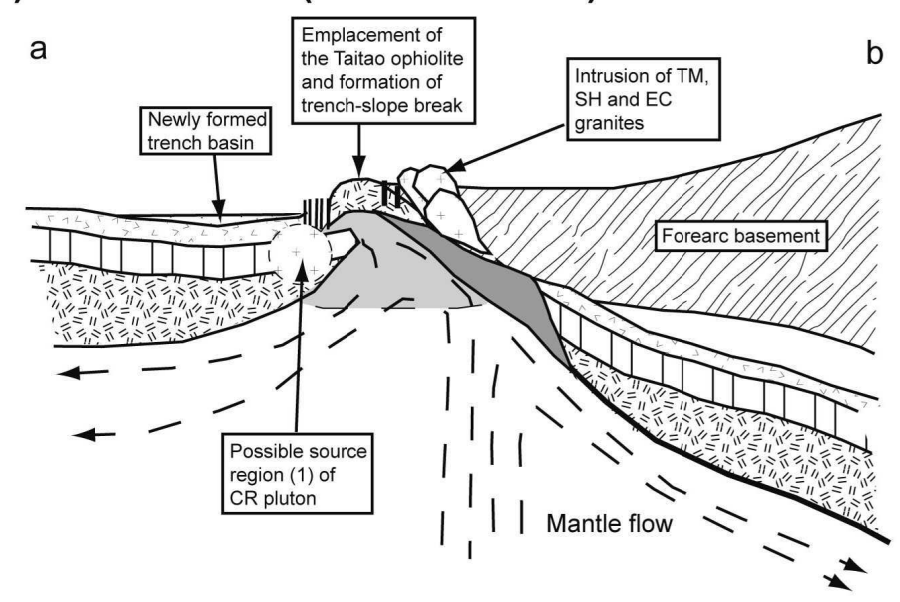

Figure 9. (a) Migration and subduction of the Chile ridge at $8 \mathrm{Ma}$. (b) Emplacement of the Taitao ophiolite and Taitao granites at $~ 6$ Ma. (c) Emplacement of the Taitao ophiolite and Taitao granites at 5.7-4.6 Ma. (d) Migration and subduction of the Chile ridge at $4 \mathrm{Ma}$. (e) Cross section of ophiolite and granite emplacement at 5.7$5.1 \mathrm{Ma}$; location in (b). Figures are modified from Anma et al. (2006, 2013). with tonalitic, trondhjemitic or granodioritic compositions (Beard and Lofgren, 1990; Rapp et al., 1991; Patino Douce, 1995; Rapp and Watson, 1995; Springer and Seck, 1997; Sisson et al., 2005). The juvenile magmas that constitute the principal component of the Taitao granites, with unfractionated HREE patterns and low $\mathrm{La} / \mathrm{Yb}$ ratios, could be explained by partial melting of amphibolite in the absence of garnet (Kon et al., 2013).

The high $\mathrm{K}$ content and lower $\mathrm{MgO}$ and $\mathrm{CaO}$ contents in the Seno Hoppner granite imply partial melting of oceanic crust at a higher temperature than other plutons. This temperature contrast is reasonable because the 5.2 Ma Seno Hoppner pluton is farther from the trench, hence the subducting slab underneath it was deeper and hotter than was the case for the 5.2 Ma Estero Cono pluton and the 5.7 Ma Tres Montes pluton near the trench. Zircon saturation temperatures (Hanchar and Watson, 2003) calculated for the granites support the higher temperatures (over $810^{\circ} \mathrm{C}$ ) for the Seno Hoppner granite compared to the others (down to $770^{\circ} \mathrm{C}$ ). Partial melting of K-rich basaltic rocks of the Chile Ridge (Klein and Karsten, 1995; Sherman et al., 1997) could have formed such granitic melts (Sisson et al., 2005). Nevertheless, producing high-K granitic melts with $74 \%$ $\mathrm{SiO}_{2}$ content is not easy and may require further processing. The Seno Hoppner granite has a prominent negative $\mathrm{Eu}$ anomaly that implies fractional crystallization by settlement of Ca-plagioclase, whereas the hypothesized source gabbros with N-MORB composition have positive Eu anomalies. Considering that REE concentrations in our samples were about 10 times greater in granite than in gabbro, the granitic melts must have collected material from a vast amount (10 times their volume) of gabbro that was continually brought into the subduction zone with the moving plate.

Lagabrielle et al. (2003) emphasized the importance of repeated passages of several ridge segments to develop high thermal gradients that allow partial melting of oceanic lithosphere at temperatures of $800-900^{\circ} \mathrm{C}$ and low pressures corresponding to depths of 10 $20 \mathrm{~km}$, and to form Quaternary calc-alkaline acidic magmatism in the Chile triple junction area. It is noteworthy that the I-type calc-alkaline magmatism took place during the first ridge subduction event (Fig. 9a, b).

\section{Implication for ophiolite tectono- magmatism}

The tectonomagmatic processes we report here, especially the formation of the calc-alkaline granites at this ridge-trench intersection environment, have important implications for constructing tectonomagmatic models of ophiolites. Presence of the calcalkaline granites is usually interpreted as a suprasubduction zone signature. Our study shows that the 
(a)

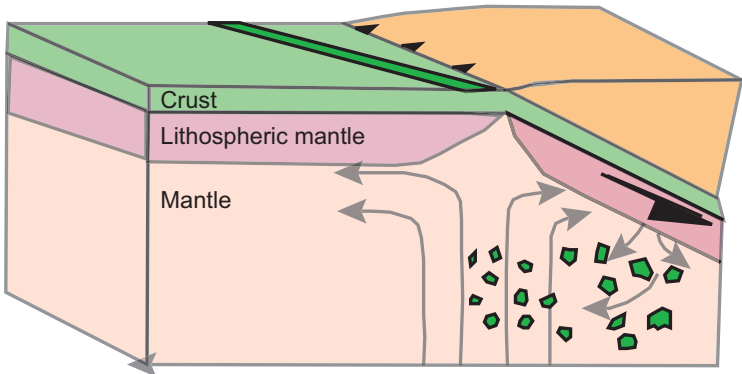

(b)

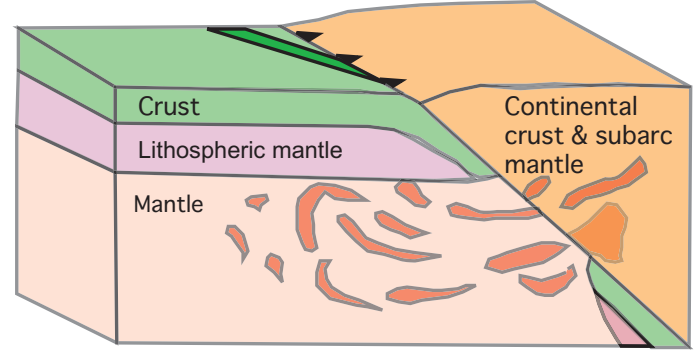

(c)

$$
\begin{aligned}
& \text { Chile trench filled by } \\
& \text { sediments derived through }
\end{aligned}
$$

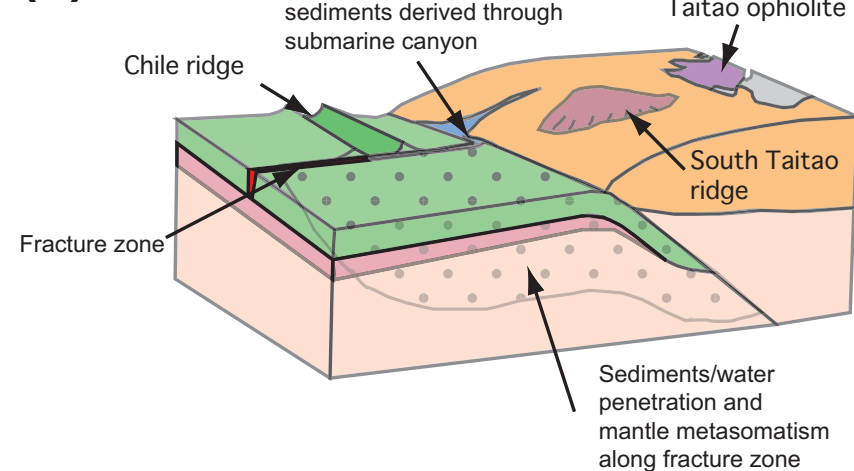

Figure 10. Models for the contamination of oceanic mantle by continental material (a) through incorporation of foundered slab material (Strum et al., 2000), (b) through a slab window connecting sub-arc mantle and sub-ridge mantle (Strum et al., 2000), and (c) through incorporation of seawater and sediment penetrating a fracture zone (this study).

calc-alkaline magmas might be generated at mid-ocean ridge segments that are about to be subducted.

As exemplified in introduction, the Taitao ophiolite-granite complex has a full range of chemical variations that is comparable to the Semail ophiolite, which had a rather prolonged tectonomagmatic history. In the case of the Semail ophiolite, the history from the generation of ophiolitic rocks to magmatism and modification at an arc environment is supported by age constraints. However, if such history relies only on geochemical signatures, then it may result in misinterpretation of the rock record of many ophiolites by mistaking what are essentially of mid-oceanic segment origin for an apparent supra-subduction zone signature.

Compared to the Semail ophiolite, the Taitao ophiolite-granite complex has a significantly greater fraction of granitic rocks. Previous have argued for for heating of the forearc region as evidenced by high geothermal gradient metamorphism in this region (Lagabrielle et al., 2000; Shibuya et al., 2007; Kon et al., 2013). Such evidence for a high geothermal gradient is lacking in the generally cold settings of emplacement for ophiolites such as Semail (Wakabayashi et al., 2010), with minor exception of the metamorphic sole that indicates a ridge subduction origin. Perhaps, the amount of calc-alkaline granites incorporated into ophiolites depends on whether the concerned oceanic lithosphere was young and hot: in the case of Semail with a prolonged history, the place of origin of ophiolitic rocks and the obducted margin were far enough such that the Cretaceous oceanic lithosphere was cooled sufficiently before obduction. This process might have resulted in a distinctive arc-type magmatism and a less amount of granitic rocks.

\section{Summary}

The late Miocene - early Pliocene Taitao ophiolite provides an excellent opportunity to study the emplacement mechanism of a ridge-trench intersection ophiolite and the complex magmatic interactions between the subducting ridge, overlying crust and sediments, and mantle. The MORB erupted near the ridge subduction zone appears to have been contaminated by a lower crustal material that has experienced partial melting. Such material could have been delivered to the sub-ridge mantle through a slab window from the sub-arc mantle, or it could have originated from sub-oceanic lithospheric mantle that had foundered during subduction and been brought to the spreading ridge by mantle upwelling. Alteration of mantle material by seawater along fracture zones and contamination by fracture zone-fill sediments are other important factors that may control magmatic interactions in a ridge-trench intersection environment. As a result of ridge subduction, juvenile magmas of calc-alkaline I-type granites were formed by partial melting of young and hot oceanic ridge lithosphere under garnet-free-amphibolite conditions. The magmas were then contaminated by subducted sediment or upper crustal components. Fractional crystallization of Ca-plagioclase favored production of granitic melt with high $\mathrm{K}_{2} \mathrm{O}$ and $\mathrm{SiO}_{2}$ contents.

\section{Acknowledgements}

This work was supported by grant-in-aid for Science Research 13373004 and $15 \mathrm{H} 02630$ by the Ministry of Education, Culture, Sports, Science and Technology of Japan. RA thanks Y. Kaneko (Meisei University), M. Terabayashi (Kagawa University), T. Komiya (University of Tokyo), S. Kagashima (Yamagata University), I. Katayama (Hiroshima University), F. Herve (University of Chile), T. Nishimura (NICHIMAR), S. Maruyama (TiTech), T. Ota (Okayama University), E. E. Veloso (Universidad Catolica del Norte), M. Schilling (Universidad Austral de Chile), S. Yamamoto (TiTech), Y. Kon (AIST), and T. Shibuya (JAMSTEC) for their field support. Our thanks go to J. Wakabayashi of California State University and an anonymous reviewer for the careful review and constructive ideas that helped to improve the manuscript. We thank Y. Dilek of Miami University for the opportunity to publish this paper and for his editorial help and improvements.

\section{References}

Adachi, Y. and Miyashita, S., 2003, Geology and petrology of the plutonic complexes in the Wadi Fizh area: Multiple magmatic events and segment structure in the northern Oman ophiolite: Geochemistry Geophysics Geosystems, v. 4, doi: 10. 1029/2001GC000272. 
Anma, R. and Orihashi, Y., 2013, Shallow-depth melt eduction due to ridge subduction: LA-ICPMS U-Pb igneous and detrital zircon ages from the Chile Triple Junction and the Taitao Peninsula, Chilean Patagonia: Geochemical Journal, v. 47, pp. 149-165.

Anma, R., Veloso-Espinosa, E. A. and the CHRISTMASSY Project-Taitao Ophiolite Study Group, 2005, Tectonic Evolution of the Taitao ophiolitean ophiolite formed by ridge collision event: Gekkan Chikyu, v. 312, pp. 428-437. (in Japanese)

Anma, R., Armstrong, R., Danhara, T., Orihashi, Y. and Iwano, H., 2006, Zircon sensitive high mass-resolution ion microprobe $\mathrm{U}-\mathrm{Pb}$ and fissiontrack ages for gabbros and sheeted dykes of the Taitao ophiolite, Southern Chile, and their tectonic implications: Island Arc, v. 15, pp. 130-142.

Anma, R., Armstrong, R., Orihashi, Y., Ike, S., Shin, K-C., Kon, Y., Komiya, T., Ota, T., Kagashima, S., Shibuya, T., Yamamoto, S., Veloso, E. E., Fannin, M. and Herve, F., 2009, Are the Taitao granites formed due to subduction of the Chile ridge? Lithos, v. 113, pp. 246-258.

Anonymous, 1972, Penrose field conference on ophiolites: Geotimes, v. 17, pp. 24-25.

Balestro, G., Festa, A., Dilek, Y. and Tartarotti, P., 2015, Pre-Alpine Extensional tectonics of a peridotite-localized oceanic core complex in the late Jurassic, high-pressure Monviso ophiolite (Western Alps). Episodes, v. 38, No. 4, pp. 266-282, doi:10.18814/epiiugs/2015/v38i4/82421.

Barker, F., 1979, Trondhjemite: definition, environment and hypothesis of origin: in Baker, F. (ed), Trondhjemite, Dacite and Related Rocks: Development in Petrology, v. 6, pp. 1-12.

Beard, J.S. and Lofgren, G.E., 1990, Dehydration melting and water-saturated melting of basaltic and andestic greenstones and amphibolites at 1, 3, and $6.9 \mathrm{~kb}$ : Journal of Petrology, v. 32, pp. 365-401.

Behrmann, J. H., Lewis, S. D., Cande, S. C. and ODP Leg 141 Scientific Party, 1994, Tectonic and geology of spreading ridge subduction at Chile Triple Junction: a synthesis of results from Leg 141 of the Ocean Drilling Program: Geologische Rundschau, v. 83, pp. 832-852.

Boudier, F. and Nicolas, A., 2011, Axial melt lenses at oceanic ridges - A case study in the Oman ophiolite: Earth and Planetary Science Letters, v. 304, pp. 313-325

Boudier, F., Nicolas, A. and Ildefonse, B., 1996, Magma chambers in the Oman ophiolite: fed from the top and the bottom: Earth and Planetary Science Letters, v. 304, pp. 313-325.

Bourgois, J., Lagabrielle, Y., Maury, R., Le Moigne, J., Vidal, P., Cantagrel, J. M. and Urbina, O., 1992, Geology of the Taitao peninsula (Chile margin triple junction area, $46-47^{\circ} \mathrm{S}$ ); Miocene to Pleistocene obduction of the Bahia Barrientos Ophiolite: EOS, v. 73, (43) 592.

Bourgois, J., Lagabrielle, Y., Le Moigne, J., Urbina, O., Janin, M-C., Beuzart, P., 1993, Preliminary results of a field study of the Taitao ophiolite (southern Chile): Implications for the evolution of the Chile triple junction: Ofioliti, v. 18, pp. 113-129.

Bourgois, J., Martin, H., Lagabrielle, Y., Le Moigne, J. and Frutos Hara, J., 1996, Subduction erosion related to spreading-ridge subduction: Taitao peninsula (Chile margin triple junction area): Geology, v. 24, pp. 723726.

Breitsprecher, K. and Thorkelson, D. J., 2009, Neogene kinematic history of Nazca-Antarctica-Phoenix slab windows beneath Patagonia and the Antarctic Peninsula: Tectonophysics, v. 464, pp. 10-20.

Cande, S. and Leslie, B., 1986, Late Cenozoic tectonics of southern Chile trench: Journal of Geophysical Research, v. 91 (B1), pp. 471-96.

Cande, S., Herron, E. and Hall, B., 1982, The early Cenozoic tectonic history of the southeast Pacific: Earth Planetary Science Letters, v. 57, pp. 63-74.

Cande, S. C., Leslie, R. B., Parra, J. C. and Hobart, M., 1987, Interaction between the Chile ridge and Chile trench: Geophysical and geothermal evidence: Journal of Geophysical Research, v. 92 (B1), pp. 495-520.

Chappell, B. W. and White, A. J. R., 1974, Two contrasting granite types: Pacific Geology, v. 8, pp. 173-174.

Dilek, Y. and Newcomb, S., 2003, Ophiolite concept and the evolution of geological thought: The Geological Society of America Special Paper, vol. 373, ISBN 0-8137-2373-6.

Dilek, Y. and Flower, M.F.J., 2003, Arc-trench rollback and forearc accretion: 2. A model template for ophiolites in Albania, Cyprus, and Oman: The Geological Society of London Special Publications, v. 218, pp. 43-68.
Dilek, Y. and Robinson, P.T., 2003, Ophiolites in Earth history. Geological Society, London, Special Publication, v. 218, pp. 1-8.

Dilek, Y. and Furnes, H., 2009, Structure and geochemistry of Tethyan ophiolites and their petrogenesis in subduction rollback systems: Lithos, v. 113, pp. 1-20.

Dilek, Y. and Furnes, H., 2011, Ophiolites genesis and global tectonics: Geochemical and tectonic fingerprinting of ancient oceanic lithosphere: Geological Society of America Bulletin, v. 123, pp. 387-411.

Dilek, Y. and Furnes, H., 2014, Ophiolites and their origins: Elements, v. 10, pp. 93-100.

Fareeduddin and Dilek, Y., 2015, Structure and petrology of the NagalandManipur Hill ophiolite melange zone, NE India: A fossil Tethyan subduction channel at the India-Burma plate boundary. Episodes, v. 38, No. 4, pp. 298-314, doi:10.18814/epiiugs/2015/v38i4/82426

Flower, M.J.F, and Dilek, Y., 2003, Arc-trench rollback and forearc accretion: 1. A collision-induced mantle flow model for Tethyan ophiolites: The Geological Society of London Special Publications 2003, v. 218, pp. 2141.

Forsythe, R., Nelson, E., Carr, M., Kaeding, M. E., Herve, M., Mpodozis, C., Soffia, J. M. and Harambour, S., 1986, Pliocene near-trench magmatism in southern Chile: A possible manifestation of the ridge collision. Geology, v. 14 , pp. 23-27.

Guivel, C., Lagabrielle, Y., Bourgois, J., Maury, R. C., Fourcade, S., Martin, H. and Arnaud, N., 1999, New geochemical constraints for the origin of ridge-subduction-related plutonic and volcanic suites from the Chile triple junction (Taitao peninsula and Site 862, LEG ODP141 on the Taitao ridge): Tectonophysics, v. 311, pp. 83-111.

Hanchar, J. M. and Watson, E. B., 2003, Zircon Saturation Thermometry: Reviews in Mineralogy and Geochemistry, v. 53, pp. 89-112.

Herron, E. M., Cande, S. C. and Hall, B. R. (1981) An active spreading center collides with a subduction zone: A geophysical survey of the Chile Margin triple junction. Geol. Soc. Am. Memoir 154, 683-701.

Herve, F., Fanning, M. C., Thomson, S. N., Pankhurst, R. J., Anma, R., Veloso, E. E. and Herrera, C., 2003, SHRIMP U-Pb and FT Pliocene ages of near-trench granites in Taitao peninsula, Southern Chile: Short Papers IV South American Symposium on Isotope Geology, pp. 190-193.

Jacobs, J., Thomas, R. J., Ksienzky, A. K. and Dunkl, I., 2015, Tracking the Oman Ophiolite to the surface - New fission track and (U-Th)/He data from the Aswad and Khor Fakkan Blocks, United Arab Emirates: Tectonophysics, v. 644-645, pp. 68-80.

Kaeding, M., Forsythe, R. and Nelson, E., 1990, Geochemistry of the Taitao ophiolite and near-trench intrusions from the Chile margin triple junction: Journal of South American Earth Science, v. 3, pp. 161-177.

Kanke, N., and Takazawa, E., 2014, A kilometer-scale highly refractory harzburgite zone in the mantle section of the northern Oman Ophiolite (Fizh Block): implications for flux melting of oceanic lithospheric mantle: Geological Society, London, Special Publications, v. 392, pp. 229-246.

Karsten, J. L., Klein E. M. and Sherman, S. B., 1996, Subduction zone geochemical characteristics in ocean ridge basalts from the southern Chile Ridge: implications of modern ridge subduction systems for the Archean: Lithos, v. 37, pp. 143-161.

Kilian, R. and Behrmann, J. H. (2003) Geochemical constraints on the sources of Southern Chile Trench sediments and their recycling in arc magmas of the Southern Andes. J. Geol. Soc., London, v. 160, pp. 57-70.

Klein, E. M. and Karsten, J. L., 1995, Ocean-ridge basalts with convergentmargin geochemical affinities from the Chile ridge: Nature, v. 374, pp. 52-57.

Kon, Y., Komiya, T., Anma, R., Hirata, T., Shibuya, T., Yamamoto, S. and Maruyama, S., 2013, Petrogenesis of the ridge subduction-related granitoids from the Taitao Peninsula, Chile Triple Junction area: Geochemical Journal, v. 47, pp. 167-183.

Lagabrielle, Y., Guivel, C., Maury, R., Bourgois, J., Fourcade, S. and Martin, H., 2000, Magmatic-tectonic effects of high thermal regime at the site of active ridge subduction: The Chile triple junction model. Tectonophysics, v. 326, pp. 255-268.

Lagabrielle, Y., Le Moigne, J., Maury, R., Cotton, J. and Bourgois, J., 1994, Volcanic record of the subduction of an active spreading ridge, Taitao peninsula (southern Chile): Geology, v. 22, pp. 515-518. 
Le Maitre, R. W., Streckeisen, A., Zanettin, B., Le Bas, M. J., Bonin, B., Bateman, P., Bellieni, G., Dudek, A., Efremova, S., Keller, J., Lameyre, J., Sabine, P. A., Schmid, R., Sorensen, H. and Woolley, A. R., 2005, Igneous Rocks: A Classification and Glossary of Terms ( $2^{\text {nd }}$ Edition): Cambridge University Press, Cambridge, 256 pp.

Le Moigne, J., Lagabrielle, Y., Whitechurch, H., Girardeau, J., Bourgois, J. and Maury, R. C., 1996, Petrology and geochemistry of the ophiolitic and volcanic suites of the Taitao peninsula-Chile triple junction area: Journal of South American Earth Science, v. 9, pp. 43-58.

MacLeod, C. J. and Yaouancq, G., 2000, A fossil melt lens in the Oman ophiolite: Implications for magma chamber processes at fast spreading ridges: Earth and Planetary Science Letters, v. 176, pp. 357-373.

McDonough, W. F. and Sun, S.-S., 1995, The composition of the Earth: Chemical Geology, v. 120, pp. 223-225.

Miyashiro, A., 1973, The Troodos ophiolitic complex was probably formed in an island arc: Earth and Planetary Science Letters, v. 19, pp. 218-224.

Miyashita, S., Adachi, Y. and Umino, S., 2003, Along-axis magmatic system in the northern Oman ophiolite: Implications of compositional variation of the sheeted dike complex: Geochemistry Geophysics Geosystems, v. 4, doi: 10. 1029/2001GC000235.

Mpodozis, C., Herve, M., Nasi, C., Soffia, J., Forsythe, R. and Nelson, E., 1985, El magmatismo plioceno de la península Tres Montes y su relación con la evolución del punto triple de Chile: Revista Geolgica de Chile, v. 25-26, pp. 13-28.

Nelson, E., Forsythe, R., Diemer, J., Allen, M. and Urbina, O., 1993, Taitao ophiolite: A ridge collision ophiolite in the forearc of southern Chile (46 $\left.{ }^{\circ} \mathrm{S}\right)$ : Revista Geolgica de Chile, v. 20, pp. 137-166.

Orihashi, Y. and Hirata, T., 2003, Rapid quantitative analysis of Y and REE abundances in XRF glass bead for selected GSL reference rock standards using Nd-YAG $266 \mathrm{~nm}$ UV laser ablation ICP-MS: Geochemical Journal, v. 37 , pp. $401-412$.

Patino Douce, A.E., 1995, Experimental generation of hybrid silicic melts by reaction of high-Al basalt with metamorphic rocks: Journal of Geophysical Research, v. 100 (B8), pp. 15623-15639.

Pearce, J. A. and Robinson, P. T., 2010, The Troodos ophiolitic complex probably formed in a subduction initiation, slab edge setting: Gondwana Research, v. 18, pp. 60-81.

Rapp, R.P., Watson, E.B. and Miller, C.F., 1991, Partial melting of amphibolite/ eclogite and the origin of Archean trondhjemites and tonalities: Precambrian Research, v. 51, pp. 1-25.

Rapp, R.P. and Watson, E.B., 1995, Dehydration melting of metabasalt at 832 kbar: implications for continental growth and crust-mantle recycling: Journal of Petrology, v. 36, pp. 891-931.

Saccani, E., Dilek, Y., Marroni, M., and Pandolfi, L., 2015, Continental margin ophiolites of Neotethys: Remnants of ancient Ocean-Continent Transition Zone (OCTZ) lithosphere and their geochemistry, mantle sources and melt evolution patterns. Episodes, v. 38, No. 4, pp. 230-249, doi:10. 18814/epiiugs/2015/v38i4/82418.

Schulte, R. F., Schilling, M., Anma, R., Farquhar, J., Horan, M., Komiya, T., Piccoli, P. M., Pitcher, L. and Walker, R., 2009, Chemical and chronologic complexity in the convecting upper mantle: Evidence from the Taitao ophiolite, southern Chile: Geochimica Cosmochimica Acta, v. 73, pp. 5793-5819.

Sherman, S. B., Karsten, J. L. and Klein, E. M., 1997, Petrogenesis of axial lavas from the southern Chile ridge: major element constraints: Journal of Geophysical Research, v. 102, B7, pp. 14963-14990.

Shibuya, T., Komiya, T., Anma, R., Ota, T., Omori, S., Kon, Y., Yamamoto, S. and Maruyama, S., 2007, Progressive metamorphism of the Taitao ophiolite; Evidence for axial and off-axis hydrothermal alterations: Lithos, v. 98, pp. 233-260.

Sisson, T.W., Ratajeski, K., Hankins, W.B., Glazner, A.F., 2005, Voluminous granitic magmas from common basaltic sources: Contributions to Mineralogy and Petrology, v. 148, pp. 635-661.

Springer, W. and Seck, H.A., 1997, Partial fusion of basic granulites at 5 to 15 kbar: implications for the origin of TTG magmas: Contributions to Mineralogy and Petrology, v. 127, pp. 30-45.

Strum M. E., Klein, E. M., Graham, D. W. and Karsten, J., 1999, Age constraints on crustal recycling to the mantle beneath the southern Chile
Ridge: He-Pb-Sr-Nd isotope systematics: Journal of Geophysical Research, v. 104, B3, pp. 5097-5114.

Strum M. E., Klein, E. M., Karsten, J. and Karson, J. K., 2000, Evidence for subduction-related contamination of the mantle beneath the southern Chile Ridge: Implications for ambiguous ophiolite compositions: In, Dilek, Y. and others (Editors), Geological Society of America, Special Paper, v. 349, pp. 13-20.

Tani, K., Orihashi, Y. and Nakada, S., 2002, Major and trace component analysis of silicate rocks using fused glass bead by X-ray Fluorescence spectrometer: Evaluation of analytical precision for third, sixth and eleventh dilution fused glass beads. Technical Research Report, Earthquake Research Institute, University of Tokyo, v. 8, pp. 26-36.

Taylor, S. R. and McLennan, S. M., 1985, The Continental Crust: Its Composition and Evolution: Blackwell, Oxford, $312 \mathrm{pp}$.

Tebbens, S. F. and Cande, S. C., 1997, Southeast pacific tectonic evolution from early Oligocene to Present: Journal of Geophysical Research, v. 102, pp. 12061-12084.

Tebbens, S. F., Cande, S. C., Kovacs, L., Parra, J. C., LaBrecque, J. L. and Vergara, H., 1997, The Chile ridge: A tectonic framework: Journal of Geophysical Research, v. 102, pp. 12035-12059.

Thomson, S. N. and Herve, F., 2002, New time constraints for the age of metamorphism at the ancestral Pacific Gondowana margin of southern Chile $\left(42^{\circ}-52^{\circ} \mathrm{S}\right)$ : Revista Geologica de Chile, v. 29, pp. 255-271.

Tsuchiya, N., Shibata, T., Yoshikawa, M., Adachi, Y., Miyashita, S., Adachi, T., Nakano, N. and Osanai, Y., 2013, Petrology of Lasail plutonic complex, northern Oman ophiolite, Oman: An example of arc-like magmatism associated with ophiolite detachment: Lithos, v. 156-159, pp. $120-138$.

Veloso, E. E., Anma, R. and Yamazaki, T., 2005, Tectonic rotations during the Chile ridge collision and obduction of the Taitao ophiolite (Southern Chile): Island Arc, v. 14, pp. 599-615.

Veloso, E. E., Anma, R., Ota, T., Komiya, T., Kagashima, S. and Yamazaki, T., 2007, Paleocurrent patterns of the sedimentary sequence of the Taitao ophiolite constrained by anisotropy of magnetic susceptibility and paleomagnetic analyses. Sedimentary Geology, v. 201, pp. 446460.

Veloso, E. E., Anma, R. and Yamaji, A., 2009, Ophiolite emplacement and the effects of the subduction of the Chile Ridge System: Heterogeneous paleostress regimes recorded in the Taitao ophiolite (Southern Chile): Andean Geology, v. 36, pp. 3-16.

Wakabayashi, J., Ghatak, A. and Basu, A. R., 2010, Tectonic setting of suprasubduction zone ophiolite generation and subduction initiation as revealed through geochemistry and regional field relationships: Geological Society of America Bulletin, v. 122, pp. 1548-1568.

Wakabayashi, J. and Dilek, Y., 2003, What constitutes 'emplacement' of an ophiolite?: mechanism and relationship to subduction initiation and formation of metamorphic soles: Geological Society, London, Special Publications, v. 218, pp. 427-447.

Warren, C. J., Parrish. R. R., Waters, D. L. and Seale, M. P., 2005, Dating the geologic history of Oman's Semail ophiolite: insights from U-Pb geochronology: Contribution to Mineralogy and Petrology, v. 150, pp. $403-422$.

Wasserburg, G.J., S.B. Jacobsen, D.J. DePaolo, M.T. McCulloch, and T. Wen, 1981, Precise determination of $\mathrm{Sm} / \mathrm{Nd}$ ratios, $\mathrm{Sm}$ and $\mathrm{Nd}$ isotopic abundances in standard solutions: Geochimica Cosmochimica Acta, v. 45, pp. 2311-2323.

Yamato, P., Agard, P., Goffe, B., De Andrade, V., Vidal, O. and Jolivet, L., 2007, New, high-precision P-T estimates for Oman blueschists: implications for obduction, nappe stacking and exhumation processes: Journal of Metamorphic Geology, v. 25, pp. 657-682.

Yang, G.X. and Dilek, Y., 2015, OIB- and P-Type ophiolites along the YarlungZangbo Suture Zone (YZSZ), southern Tibet: Polyphase melt history and mantle sources of the Neotethyan oceanic lithosphere. Episodes, v. 38 , No. 4 , pp. 250-265, doi:10.18814/epiiugs/2015/v38i4/82420.

Yoshikawa, M., Python, M., Tamura, A., Arai, S., Takazawa, E., Shibata, T., Ueda, A. and Sato, T., 2015, Melt extraction and metasomatism recorded in basal peridotites above the metamorphic sole of the northern Fizh massif, Oman ophiolite: Tectonophysics, v. 650, pp. 53-64. 